\title{
Altered Microbiota Composition Mediates Depressive Behavior during Chronic Stress
}

\author{
Ioana Alexandra Marin \\ Galati, Romania
}

B.S. Neuroscience, Lafayette College, 2011

A Dissertation presented to the Graduate Faculty of the University of Virginia in Candidacy for the Degree of Doctor of Philosophy

Neuroscience Graduate Program

University of Virginia

May 2017 
(C) 2017 Ioana A. Marin 


\section{Acknowledgements and Thanks}

In the most direct sense, this research work would not have been possible without my mentors, Jony Kipnis and Alban Gaultier, as well as our funding sources: the Office of the UVA Vice President for Research and the National Institute for Mental Health. Jony was really the first one to capitalize on my interest in how the gut influences mood and behavior by encouraging me to write several preliminary grants for a funding opportunity issued by the Vice President's Office. Again at Jony's encouragement, I reached out for collaborators across the University, who have also been instrumental to this research, both technically and with guidance. Such collaborators include Martin Wu and his team in the Biology Department, and Suna Onengut-Gumuscu and Emily Farber in the Center for Public Health Genomics. Soon after I started working on this project, Alban became instrumental for his expertise in molecular biology and biochemistry. This research has been a truly collaborative effort and I would like to thank everyone who made it possible and guided me to navigate through three systems of biology.

I would also like to acknowledge the current members of my committee: Iggy Provencio, Michael Scott, Bill Petri, John Lukens, as well as past members (Patrice Guyenet, Bettina Winkler and Ali Guler) for their advice and critics that helped made my science more rigorous.

Finally, my undergraduate student, Jennifer Goertz, has been instrumental in assisting with some of the experiments.

The above-mentioned individuals, as well as many others, are due thanks for contributing to my scientific and personal growth during my Ph.D. training. I will try to recognize them below.

I would first like to recognize my first adviser, Jony, for his infectious passion for progressive science. Jony drew me into his group by presenting me with a question I had never thought of before and throughout the years I have seem him pose such questions many times. To dream of things that never were and then try to pursue them takes real talent and dedication. I hope some of it rubbed off on me. I am grateful to Jony for gathering such amazing individuals as I have been working with in the many iterations of the lab and allowing me to follow my own path among them. Jony provided great opportunities during my PhD, including meeting amazing scientists and support for attending many great conferences. Perhaps most importantly, I am grateful for the sharp questions and timely criticism, which not only made me a more thorough scientist, but also taught me to continuously consider the relevance of the questions I ask and the experiments I perform.

Also first, I ought to recognize my second (acquired) adviser, Alban. One day, I went to Alban for help troubleshooting a sensitive PCR reaction and from then on, the trips to his office increased logarithmically. Alban's constant optimism and excitement for doing science is inspiring, particularly during the hardest times. His ability to break complex questions down to simple ones has helped me to both plan better experiments and analyze more critically. Alban has a wealth of technical tricks that he pulls from up his sleeve just when needed and an amazing talent to find in 1-2 minutes information I spent hours looking for. Above all, I am grateful to Alban for his dedication, availability, and constant willingness to think and plan experiments and ideas with me. 
Several friends in the lab have been instrumental in my training as a scientist. Big thanks go to: Noel Derecki for teaching me many many techniques as well as always asking me what the hypotheses behind my experiments were; Sachin Gadani for all the joint efforts early on in the lab and many "Sunday" experiments; Jim Cronk for teaching me the importance of counting how many time I triturate or mix any cell suspension; Antoine Louveau for a great example of a tasteful approach to science. To every member of the Kipnis and Gaultier labs: thank you for your honest feedback, questions and sharing your inspiration. It's been an honor to learn from and along with each of you.

Thank you to my college research adviser, Lisa Gabel, for her continuous support and guidance. To everyone in the lab and in the greater BIG Center: thank you for the laughs, collegiality and willingness to help.

A PhD is not just a time of scientific growth. By the nature of the scientific method, as well as our imperfect approaches, it is a time riddled with both highs and lows. This rollercoaster ride thus ends up also being a feat of mental strength and a period of personal growth (hopefully!). So I must also acknowledge everyone who supported me on this front. Friends from high-school (Sinzi and Elena) and college (Nafis, Joaquin, Pooja, Brook, Hannah, Farah, Farai, Elliott, Ahsan, Basu) who stood to remind me of my strengths, beliefs, and abilities - thank you for the talks, the visits, the messages. Friends in Charlottesville - thank you for climbing mountains (hi Irene), sharing Thanksgiving meals, and being my community. Sachin, thanks for making bugs a little less scary, for letting me use your car for the longest time, and for picking up the phone when I was walking late at night. A, thank you for teaching me so many things about gender and pharmacology! Antoine, thank you for all the coffee trips, that one time you cooked me mussels, and naming your T cell "Boo".

And thank you, Anthony! I love you the most. Thank you for your love, support, and our wonderful family.

I purposefully saved my family for last, because they are the most important. My mother likes an approximate quote she heard: "many generations before you have prayed so you could achieve what you achieved." I know it to be true. I am the first in my family to get a college degree, but I have been fortunate to have many of my small family teach me about perseverance and reaching higher. My grandparents have been examples of hard work, humanity, and legacy. I am grateful for giving me strong roots. Above all, however, I wouldn't have been anywhere near where I am today without my mother. She has this extraordinary ability of balancing between pushing me further and giving me confidence with very careful timing, to yield positive results. She taught me to be kind, curious, and perseverant. Though she always says I left home too young and went too far, the foundation she laid within me has always guided me. Multumesc, mama! 


\section{Table of Contents}

$\begin{array}{ll}\text { Acknowledgements and Thanks } & \text { i }\end{array}$

Part I: Making Sense of Depression $\quad 3$

Clinical aspects of depression 3

Modeling depression in animals 4

$\begin{array}{lr}\text { Neurobiology of depression } & 6\end{array}$

$\begin{array}{ll}\text { The monoamine deficiency theory } & 6\end{array}$

$\begin{array}{lr}\text { The excitatory synapse theory } & 8\end{array}$

$\begin{array}{lr}\text { The glucocorticoid theory } & 9\end{array}$

$\begin{array}{ll}\text { The circuitry of depression } & 10\end{array}$

$\begin{array}{ll}\text { Susceptibility to depression } & 13\end{array}$

An unusual candidate - the gut microbiota 15

$\begin{array}{ll}\text { Human vs mouse microbiome } & 16\end{array}$

$\begin{array}{ll}\text { Contributions of the gut microbiota to host physiology } & 17\end{array}$

$\begin{array}{ll}\text { Microbiota-immune interactions } & 18\end{array}$

Ripples of microbiome effects on the brain 20

$\begin{array}{ll}\text { Summary } & 22\end{array}$

Part II: Involvement of the Gut Microbiota in the Development and Progression of Depressive Behavior 24

Hypothesis and aims $\quad 24$ 
Chronic antibiotic treatment induces depressive behavior 25

Microbiota composition is altered by unpredictable chronic mild stress 26

Increased intestinal motility, rather than the immune system, may lead to the $\begin{array}{ll}\text { microbiota dysregulation } & 30\end{array}$

Treatment with a Lactobacillus species ameliorates despair behavior by restoring kynurenine metabolism

\section{Part III: Discussion and Future Directions 42}

Putting the data in context $\quad 42$

Open-ended questions $\quad 45$

1. Is loss of Lactobacillus sufficient to induce depressive behavior?

2. Is Lactobacillus-derived ROS the mechanism behind altered kynurenine metabolism? $\quad 46$

3. How can peripheral kynurenine modulate brain activity? 47

4. What is the role of the immune system in maintaining resilience? 49

Can gut microbiota dysregulation mediate human depression? 51

New avenues: the role of kynurenine metabolism in multiple sclerosis associated

depression $\quad 53$

$\begin{array}{ll}\text { Materials and Methods } & 57\end{array}$

$\begin{array}{ll}\text { Bibliography } & 65\end{array}$

$\begin{array}{lr}\text { Selected Publications } & 84\end{array}$ 


\section{Part I: Making Sense of Depression}

\section{Clinical aspects of depression}

Clinical depression encompasses a series of disorders, collectively characterized by "the presence of sad, empty, or irritable mood, accompanied by somatic and cognitive changes that significantly affect the individual's capacity to function" (DSM-5)1. Differences among specific types of depressive disorders are given by circumstances (e.g. postpartum depression) or presence of specific symptoms (e.g. psychosis, mania) 1. For the purpose of this dissertation, "depression" will refer to the most prevalent among this group of disorders - major depressive disorder.

A diagnosis of depression can be based on one of several depression scales (Hamilton, PHQ-9, Montgomery-Asberg), each of which rates the severity of symptoms related to mood, loss if interest/ pleasure, guilt, suicidal thoughts, sleeping and weight patterns, fatigue or agitation, as well as others (some scales are more extensive than others) ${ }^{1-3}$. Required for the diagnosis is the presence of either depressed mood or loss of interest/ pleasure, accompanied by four more other symptoms, for at least two weeks ${ }^{1}$. It is important to note that depressive disorders are diagnosed when the symptoms cannot be attributed to other physical diseases, such as multiple sclerosis, stroke etc, with known etiologies and which are known to present comorbid depression. 
Depression is one of the most prevalent mental disorders, with a lifetime prevalence of $16 \%$, in the United States, and is often accompanied by other mental disorders, such as anxiety (almost 60\%) and substance abuse (almost 25\%) ${ }^{4}$. Whether presence of such psychiatric comorbidities warrants classification of more specific types of depression has not yet been addressed by either clinicians or scientists.

\section{Modeling depression in animals}

With depression being a mental health disorder, diagnosed solely based on patientreported symptoms, it is unsurprisingly difficult to study in humans. Not only are we limited in the experimental tools available, but also the subjective nature of the disease makes it difficult to obtain reproducible results. While using laboratory animals can overcome both of these problems, their main downside is their validity in completely modeling depressive behavior.

Three main experimental paradigms are accepted as tests for depressive behavior: the forced swim test, the tail suspension test, and the sucrose preference test. The first two assays entail putting an animal in an uncomfortable situation and measuring how long it struggles to escape and/or when it ceases to struggle, with the expectation that an animal exhibiting depressive behavior will struggle less. These tests aim to measure human equivalents of despair and, since the introduction of the forced swim test in $1977^{5}$, both or either test have been used to quantify depressive behavior, as well as to screen candidate antidepressant drugs. The forced swim test is, by far, the most used test for depressive behavior, due to its increased reliability 
compared to the tail suspension test ${ }^{6}$. The sucrose preference test entails presenting an animal with a choice between regular and sweetened water and measuring its preference for the sweetened choice. The test aims to measure anhedonia, one of the core symptoms of depression, with the expectation that an animal exhibiting depressive behavior will have a decreased preference for sucrose. However, the reliability of the test is very low, with most animal models not showing decrease in their sucrose preference (perhaps due to the salience of the sweetened water $)^{6,7}$. This often leads researchers to resort to modified versions of the test, such as the sucrose consumption test, which just measures the intake of sweet water after a period of fasting. Moreover, considering that sucrose intake is a food-based behavior, its validity for measuring anhedonia can also be questioned. Many depression patients will exhibit a lack of engagement in hobbies and pleasurable activities, but may actually overeat or binge on pleasurable foods ${ }^{1-3}$. In addition to such tests that are accepted measures of depressive behavior, researchers often supplement their behavior testing with anxiety assays (such as the elevated plus maze, novelty suppressed feeding, marble burying, etc) based on the high comorbidity of anxiety and depressive disorders ${ }^{1,4,6}$.

In terms of the animal models exhibiting depressive behavior, these are generally based on environmental manipulations, the most popular of which are stress-based paradigms ${ }^{6,8}$. The most widely used assays include the chronic unpredictable stress, chronic restraint stress, repeated social defeat, and learned helplessness after unescapable foot shock. All of these models and their variants are based on the observation that stressful life events are a risk factor for developing depression ${ }^{1,9}$. This basis on repeated stress gives them limited validity, nevertheless superior to older models such as olfactory bulbectomy or corticosterone injections. Another valuable model is that of selective breeding of animals displaying high depressive 
behavior. While this paradigm aims to model the importance of a genetic predisposition for depression, it is unfortunately less widely used due to the expenses and the time requirement associated with breeding and screening outbred animals ${ }^{6,10}$.

\section{Neurobiology of depression}

Most scientists and clinicians would now agree that depression is ultimately a product of altered brain function, caused by defective circuitry and/ or chemical imbalances. This view is actually relatively young, gaining popularity following initial studies with early antidepressant drugs in the 1950s. At the time, the prevalent hypothesis was that depression "emerged from intrapsychic conditions and conflicts," the therapies of choice being psychotherapy and electric shock therapy ${ }^{11}$. In this context, perhaps unsurprisingly, the first antidepressant drugs, iproniazid and imipramine, were discovered accidentally ${ }^{11}$. Iproniazid was initially used to treat tuberculosis, while imipramine was being tested for schizophrenia, when clinicians observed their mood enhancing effects ${ }^{11-13}$.

\section{The monoamine deficiency theory}

Not only did the subsequent approval of antidepressant drugs revolutionize treatment, but also further studies on their mechanism of action provided the first neurobiological theory of depression: the monoamine deficiency theory, which remains one of the fundamental theories of depression ${ }^{9,11,14}$. This theory postulates that depression is caused by a general deficit in monoamine neurotransmitters: serotonin, dopamine, norepinephrine. Interestingly, this 
theory was developed at a time when serotonin was mostly known for its peripheral effects, such as in intestinal function and blood pressure control, while its roles in the brain were still speculative ${ }^{15,16}$. Yet, the success of these early antidepressants led to rapid expansion of the tricyclic family (TCAs), followed by the selective serotonin reuptake inhibitors (SSRIs, starting in the 1970s), and other atypical neurotransmitters, all aimed at boosting amounts of brain amine neurotransmitters (serotonin, dopamine, norepinephrine) ${ }^{11,14}$. There is no doubt that antidepressant compounds can affect brain function and influence behavior in both positive (mood elevation, anxiolysis) and negative (many peripheral side effects) ways. However, whether they correct an underlying dysregulation is debatable and now beginning to be challenged $14,17,18$. In fact, evidence that depressed patients have a deficiency in brain levels of any of the monoamines is very scarce. Studies trying to reduce amine neurotransmitter levels by acute depletion of their amino acid precursors report a fast induction of negative affect only in patients with a history of depression ${ }^{19}$. Moreover, chronic tryptophan depletion in laboratory animals has yielded inconsistent results regarding their depressive phenotype 20,21,22. In fact, many knockout animal models of enzymes involved in aminergic transmission show a depressive-resistant phenotype, rather than a pro-depressive phenotype (reviewed by Cryan and Mombereau ${ }^{6}$ ). Finally, animal studies investigating a circuitry of depression (discussed in more detail below), are now showing that both increases and decreases in the levels of released amine neurotransmitters can be observed in different brain regions in animal models of depression, indicating that it is the circuit activity that regulates neurotransmitter balance ${ }^{23-25}$. With these accumulating data, and considering the rapid raise in amine levels after antidepressant treatment, it is now believed that the delayed efficacy of the drugs is due to a progressive change in neuronal circuits due to sustained elevated levels of amines, rather than 
to correcting underlying deficits $14,26,27$. This new view would also account for the high relapse rates after treatment cessation.

\section{The excitatory synapse theory}

Unfortunately, while this drug-directed approach to seeking mechanisms of depression has proven unfruitful, scientists are still advocating for its pursuit, now based on an even broader acting drug - ketamine ${ }^{28}$. Ketamine is an NMDA receptor antagonist that was developed in the 1960s and soon approved for its use as an anesthetic ${ }^{29,30}$. In the past two decades, the drug also started receiving attention for its rapid antidepressant effects after a single administration, albeit with a transient effect ${ }^{30-32}$. Similarly to the amine-boosting antidepressant drugs, following this promising clinical success, researchers are now studying the molecular effects of ketamine administration with a view to understand mechanisms of depression ${ }^{33-35}$. Not only are these studies focusing on the acute effects of a single ketamine administration, but also to expect that the effects of a drug targeting a receptor expressed almost ubiquitously in the brain will unveil underlying causes of depression is unrealistic. Moreover, the main mechanistic readouts of ketamine effects focus on levels of BDNF. Brainderived neurotrophic factor (BDNF) is a favorite among researchers as a target-mechanism for altered neuronal processes. Early studies in the function of the molecule showed that BDNF signaling leads to increase neuronal activity via increased synaptic release and spine size ${ }^{36-39}$, which led to the view of BDNF as a generally beneficial molecule. However, more recent studies have been finding that knockout of BDNF in neuronal populations may not affect their function ${ }^{40,41}$, as well as that excess BDNF can be detrimental ${ }^{23}$. In the case of ketamine, one paper focusing on the acute effects of the drug in the hippocampus found that the drug leads 
to increased levels of BDNF ${ }^{33}$. However, a different study looking at repeated administration of ketamine (similar to what treating depression would require) found that, while ketamine acutely increases BDNF, chronically it causes a decrease in the molecule ${ }^{42}$. Therefore, making inferences about depression mechanisms based on the variable effects of a drug is, again, a dangerous strategy.

\section{The glucocorticoid theory}

Aberrant production of glucocorticoids, such as in response to repeated stressful events or early-life trauma, is another one of the most prevalent mechanisms of depression ${ }^{9,43,44}$. This theory evolved out of the observation that glucocorticoids are rapidly released under conditions of stress ${ }^{45,46}$, and that sustained high levels of glucocorticoids have detrimental effects, which include depression ${ }^{47}$. However, just like other theories involving a single molecule solution for depression, the supporting evidence is conflicting ${ }^{43,44}$. To begin with, while subsets of patients do have increased cortisol levels, or aberrant hypothalamus-pituitary-adrenal (HPA) axis activity, meta-analyses show that general associations do not hold ${ }^{43,44,48-50}$. Moreover, clinical trials with the glucocorticoid receptor blocker RU486 (Mifepristone) show that, while the drug may be effective in treating psychotic episodes, it has limited efficacy for patients with nonpsychotic unipolar depression ${ }^{51,52}$.

Animal studies on the effects of glucocorticoids have highlighted their importance for brain function and behavior. The fact that glucocorticoids are released in both stress models (i.e. forced swim) and learning tasks (i.e. morris water maze) indicates that they might actually have an adaptive role for the organism. Studies have shown that, indeed, glucocorticoid release is necessary for learning and memory ${ }^{53}$, as well as synaptic plasticity ${ }^{54,55}$. At the same time, 
both acute and chronic stress have been shown repeatedly to have negative consequences on cognitive performance ${ }^{46,56,57}$, indicating that perhaps different mechanisms than elevated glucocorticoids are responsible for the behavioral abnormalities following stress. Moreover, studies targeting the corticosterone receptors in different brain regions (both the glucocorticoid and the mineralocorticoid receptors) have shown that both knockout/down of the receptors and their overexpression leads to maladaptive, depressive-like responses ${ }^{58-62}$. These results indicate that glucocorticoid responses after stress are probably beneficial, and their balance in the brain is necessary for adaptive behaviors. Moreover, similar to the weak evidence that cortisol levels are higher in depressed patients, the evidence is also weak that corticosterone levels are elevated in chronically stressed mice ${ }^{41}$. In fact, studies show that the corticosterone release is the highest after the first episode of stress, and lower with every subsequent stress episode ${ }^{45,63}$. Given the fact that depressive behavior develops after several stress episodes, it may even be possible that the reduced corticosterone release (rather than its increase) is what contributes to depressive behavior.

\section{The circuitry of depression}

Perhaps the most recent theory of depression is that abnormal activity in a specific set of neuronal circuits yields the behavioral outputs characteristic of depression 14,28,64. The appeal of this hypothesis is that it makes sense to explain the underlying cause of depression as a standalone disorder, versus a global molecular dysregulation, which would have an array of functional manifestations, one of which would be depression. The circuitry of depression is based largely on animal studies, as clinical research is limited by the available techniques. Imaging techniques have yielded areas with different activity in depressed patients, including 
the prefrontal cortex and all areas of the limbic system ${ }^{9}$. However, such studies cannot inform about how the identified areas are wired to cause depression. Fortunately, not only are studies in laboratory animals are making progress in resolving a circuitry for depression, but also their findings largely align with the human observations 9,14,65.

Using animal models involving different types of stress protocols, as well as animals bred for congenic depressive phenotypes, studies identified deficits in reward circuitry to be associated with depressive-like behavior. Specifically, increased activity in the lateral habenula $(\mathrm{LHb})$ increases activity in the ventral tegmental area (VTA), potentiating projections to the nucleus accumbens medium spiny neurons and weakening projections to the medial prefrontal cortex (mPFC) ${ }^{65}$. Evidence is now accumulating that the habenula is acting as a "disappointment" center of the brain, with neurons in this region being excited in the absence of reward and inhibited in rewarding situations ${ }^{66-69}$. Animal models displaying depressive behavior also show increased activation of lateral habenular neurons, in turn feeding to excite the VTA ${ }^{70-72}$. Moreover, experiments involving deep brain stimulation of the habenula in both rats and humans were able to reduce neuronal activity and improve depressive symptoms ${ }^{69,70}$.

From the lateral habenula, excitatory projections potentiate the activity of dopaminergic neurons in the VTA ${ }^{70}$. In an elegant study, Krishnan and colleagues showed that potentiation of VTA neurons is specific to animals displaying depressive behavior, whereas resilient animals show compensatory mechanism that stabilize the activity of these neurons 23,73. Mechanistically, the susceptible animals show potentiation of neuronal activity in the VTA in response to repeated stress, whereas resilient animals show upregulation of inwardrectifying potassium channels $\left(\mathbf{K}_{\mathrm{ir}}\right)$, which scale back synaptic transmission ${ }^{23,73}$. What determines this difference in response to the same stimuli (repeated social defeat stress) is still 
unknown. However, studies show that overexpressing $\mathrm{K}_{\mathrm{ir}}$ channels in the VTA can correct the behavior of formerly depressive-susceptible mice ${ }^{25}$, as can optogenetically inhibiting these neurons with halorhodopsin ${ }^{24}$. Using optogenetic methods, researchers were able to show that, in the absence of stress, inhibiting neuronal activity in the VTA is aversive and induces depressive bahavior ${ }^{74}$. On the other hand, under stress conditions, which already increase neuronal activity in this region, inhibiting the VTA is beneficial, while stimulating it induces depressive-like behavior in previously resilient mice ${ }^{24,25}$. Interestingly, though the VTA projects to areas across the brain, its projections to the nucleus accumbens seem to drive much of the depressive and resilient phenotypes, with selective stimulation of these projections during stress making the difference between the two behavioral outcomes ${ }^{23,24}$.

While the past decade has shown great progress in unveiling parts of the circuitry of depression, we are still mostly understanding how mono-synaptic circuits are altered in depression animal models and whether manipulating them has any causative effects, either pro-depressive or anti-depressive. Being able to integrate these mono-synaptic circuits into a fuller "depression diagram" will be an important advance in identifying whether there is a true starting point that could trigger an altered depression circuit, or whether depression is due to generalized deficits throughout the brain, with specific modulation of certain circuits due to environmental factors (i.e. stress). For instance, hypothesizing that sustained activity in the habenula is what triggers a downstream "circuit of depression", is this sustained activity due solely to environmental inputs - such as repeated negative reinforcement? Or could there be a generalized susceptibility in the brain (such as genetic, metabolic etc), which manifests itself through depressive behavior when further challenged (i.e. with stress). Perhaps this second explanation would be more likely to account for the difference between mice resilient and 
susceptible to depressive-like behavior. Considering presumed identical genetics, rearing and exposure to stress, what would cause the selective upregulation of synaptic maintenance molecules in a subset of animals, leading to their resilient phenotype?

\section{Susceptibility to depression}

Unfortunately, the etiology of depression remains very elusive, with many possible theories and contributors. Given that the disorder is diagnosed purely based on reported symptoms, it also very likely that different underlying causes may lead to similar symptoms. Under these circumstances, one cannot talk about causes of depression, but rather discuss risk factors associated with the disorder.

The strongest association with depressive disorders is, without doubt, genetic, with a calculated heritability of depression of $37-66^{\%}{ }^{9,75}$. Despite this strong association, GWAS studies have had limited success in identifying susceptibility genes, leading to the hypothesis of complex genetic interactions accounting for an increased sensitivity to environmental factors

9. Gender is another risk factor, with women having a $70 \%$ higher lifetime prevalence of depression ${ }^{4}$. Though mechanisms for this increased susceptibility have been only sparsely investigated, this higher prevalence is attributed to both genetic and socioeconomic factors ${ }^{76}$.

In recent years, inflammation has been a factor more and more associated with depression. While the DSM-5 excludes patients with inflammatory disorders from being diagnosed with depressive disorders, the manual also acknowledges that many patients suffering of chronic inflammation experience depression symptoms ${ }^{1}$. For instance, up to half 
of the patients suffering from multiple sclerosis experience depression ${ }^{77,78}$. The exclusion of such patients from being diagnosed with depressive disorder is due to the fact that sustained inflammation induces an array of CNS symptoms in addition to depression, i.e. cognitive impairment, increased pain sensitivity, decreased locomotion ${ }^{79-81}$. Therefore, while inflammation may induce depression symptoms, the underlying neurobiology may be different from that of non-inflammatory unipolar depression. Elevated levels of cytokines, both acutely and chronically, have been extensively documented to affect synaptic transmission. I have previously reviewed such neuro-immune interactions extensively 82,83, attached in Annex A.

Environmental factors are also thought to be critical for susceptibility to depression ${ }^{1,9}$. Early life trauma or abuse, as well as a stressful lifestyle are considered the highest environmental risk factors for developing depressive disorders ${ }^{1,4,9}$. In addition, factors such as exercise and metabolism have recently been receiving a lot of attention for the roles they might play in modulating depressive symptoms ${ }^{84-87}$. Exercise has been shown to have many beneficial effects for depression outcomes in patients, with pre-clinical studies indicating that exercise can improve many aspects of physiology, from metabolism to adult neurogenesis $84,88,89$. On the other hand, in a nutrition and obesity-conscious society, nutritional imbalances are increasingly suspected of mediating or modulating depressive behaviors ${ }^{85-87,90}$. Mechanisms of how metabolism can affect brain function are just starting to be investigated ${ }^{87,91,92}$. At the same time, evidence has been mounting for a new player, the gut microbiota, as a key regulator of both metabolism and the immune system, and thus with the ability to affect the function of many organs, including the brain ${ }^{93-96}$. 


\section{An unusual candidate - the gut microbiota}

The main function of the gastrointestinal tract is digestion. Essentially a very long tube, the gut receives food, breaks it down mechanically, then enzymatically and through fermentation, absorbing nutrients and water along the way. These functions are accomplished in distinct segments of the tract, each with their own cellular specializations and accessory organs: the pancreas, liver and gall bladder for the upper GI tract, and the gut microbiota for the lower tract. The gut also possesses its own nervous system - the enteric nervous system, that develops independently from the central and peripheral nervous systems, and which is responsible for intestinal motility. Moreover, it receives extensive sympathetic and parasympathetic innervation, which controls intestinal secretions and motility ${ }^{97-100}$. While digestion in the upper tract is mostly mechanical and enzymatic, in the lower tract, bacterial fermentation is necessary for finishing the process, particularly for breaking down complex carbohydrates through fermentation, as well as for synthesizing micronutrients such as B vitamins and vitamin $\mathrm{K}{ }^{101,102}$. Though we have known about the bacterial symbioses in the intestine for a long time, it has only been recently, with the advances in sequencing technology, that we have begun to gain an appreciation for the complexity and importance of the gut microbiota beyond its function in digestion ${ }^{103,104}$.

During in utero development the fetus is sterile and bacterial colonization begins at birth when the fetus acquires a large proportion of the mother's flora ${ }^{101,105}$. Diet is one of the major regulators of the intestinal microbiota, and so the newborn flora changes with the introduction of new foods, as well as post weaning ${ }^{101,105}$. At the same time, the genetic makeup of the host also plays a role in shaping the microbiota composition ${ }^{106-108}$. While the composition of the 
microbiota is highly dynamic throughout life and dependent on external and internal factors, it is largely dominated by two bacteria phyla - the anaerobes Bacteriodetes and Firmicutes. The gram negative Bacteriodetes are primarily starch and polysaccharide degraders, whereas the gram positive Firmicutes are primarily proteolytic and amino acid fermenters 10195,102 . The presence of the abundant microbiota (up to $10^{12}$ organisms per $\mathrm{cm}^{3}$ ) is a byproduct of the slow transit through the large intestine, combined with the permissive $\mathrm{pH}$ (not as acidic as the stomach).

\section{$\underline{\text { Human vs mouse microbiome }}$}

As with many areas of biomedical science, animal studies have been instrumental in furthering our understanding of the microbiome development and function. Fortunately, the same dominant phyla are also present in mice, the laboratory model of choice in the microbiome field. Moreover, an effort comparing the microbiota composition across mouse strains to that of humans found that, functionally, the core human and mouse microbiomes are more than 95\% similar. Yet, the two species only share 4\% of microbial genomic sequences, indicating large differences in the bacterial strains they harbor 108. So far, researchers have been embracing the results found in mice, particularly considering that most microbiota studies do not have the power to taxonomically identify microbial strains, or even species. However, lowering the costs of high throughput metagenomic sequencing experiments will no doubt allow us to uncover specific functions of different strains.

Another important aspect of microbiome research is the widespread use of germ-free (GF) animals. These are animals reared in a sterile environment, as opposed to specific pathogen free (SPF), with no bacterial or fungal colonization. Studying germ-free animals has helped us 
appreciate the importance of the microbiome and the dangers of widespread antibiotic treatment. This model has also allowed scientists to selective colonize them with humanderived microbiota and further understand its function in ways that human studies do not allow 109-111. For instance, in one of the first studies of this kind, Smith and colleagues showed that it is poor diet-associated microbiota that drives growth stunting ${ }^{110}$. However, the boon of germfree mice may also lead to many misleading conclusions. Considering the many contributions of the microbiome to host physiology, missing it completely can be thought of equivalent to missing a kidney or the spleen. Though the organism can function without it, all the other systems will be affected, often by secondary effects, potentially leading to over-interpretation regarding the direct functions of the organ. Moreover, in studies involving "humanizedmicrobiome" mice, it is likely that the genetic differences and restricted diet of the mice alters the human inoculum they receive ${ }^{109,112}$. Unfortunately, most studies do not check for such differences, drawing conclusions that may not actually pertain to the initial hypothesis ${ }^{109}$. It is, therefore, important to exercise caution when using such reductionist animal models ${ }^{112}$.

\section{Contributions of the gut microbiota to host physiology}

As previously discussed, the main contribution of the gut microbiota is to host metabolism 95,102. Commensal bacteria not only help break down carbohydrates, but also, through their metabolism, release modulatory molecules. Perhaps the most widely studied compounds are short chain fatty acids, which are absorbed by the host and further impact the metabolic output of the liver ${ }^{95}$. Another direct role of the microbiota is in preventing pathogenic bacterial infections ${ }^{113}$. While a part of this function is accomplished by the simple competition for space and resources, another important mechanism is by the production of antimicrobial compounds 
that bacteria themselves synthesize and secrete ${ }^{113}$. Finally, colonization of the intestinal tract is now accepted as a requirement for the maturation and function of the immune system ${ }^{96,114-}$

116. In addition to its importance for pathogen defense, this interaction further impacts the entire organism, as it can modulate clinical outcomes such as allergy, autoimmunity or immunodeficiency.

\section{$\underline{\text { Microbiota-immune interactions }}$}

The relationship between the intestinal microbiota and the immune system is a very intimate one. The immune system is necessary for maintaining the microbiota, and the microbiota allows the immune system to mature and function normally ${ }^{96}$. At first sight, the two systems are spatially segregated: the bacteria reside in the lumen of the gut, separated by the mucus layer from the epithelial cells, and the closest immune cells are in the lamina propria (Fig. 2) ${ }^{96}$. However, interspersed between the epithelial cells are projections from dendritic cells that sample antigen from the bacteria present in the gut. The antigen is internalized and presented to B cells in the gut associate lymphoid tissue (GALT) or mesenteric lymph nodes to stimulate specific IgA production, which is then returned to the luminal surface ${ }^{117}$. In addition, innate lymphoid cells that reside in the lamina propria also stimulate secretion of antimicrobial compounds (such as RegIII $\gamma$ and S100A9) via their production of IL-22 ${ }^{118}$. While these are some of the mechanisms currently known to prevent bacteria from invading the host, a large number of studies also amount to show that the immune system can control the composition of the microbiota, perhaps by exerting specific effects on certain bacterial classes. For example, mice deficient in the inflammasome molecule NLRP6 present an altered microbiota composition enriched in Prevotellacea, that induces gut pathology ${ }^{119}$. Similar results have been 
shown with mice deficient in the pattern recognition receptor TLR5, which recognizes bacterial flagellin ${ }^{120}$, or mice deficient in the $\mathrm{T}$ cell differentiation factor Tbet ${ }^{121}$. The exact mechanisms of how these molecules (or the cell types they are expressed on) control bacterial composition remain to be investigated.

On the other hand, the presence and composition of the microbiota is necessary for normal immune system function. While the fact that germ-free mice have impaired immune function has been appreciated for years ${ }^{122}$, it has only been recently that significant studies started accumulating ${ }^{96}$. Works from the group of Dennis Kasper show that not only is intestinal colonization important, but also the composition of the microbiota is important for proper maturation of the immune system ${ }^{123,124}$. The best characterized phenotype in germ free mice is their decreased numbers of intestinal (and possibly systemic) $\mathrm{T}$ cells and the impairment of the $\mathrm{T}$ cells in maturing and acquiring memory phenotypes ${ }^{123,124}$. Perhaps as a compensatory mechanism, these mice have a much larger population of colonic natural killer T cells (NKTs), which predisposes them to pathology ${ }^{125}$. Interestingly, even though mouse, rat, and human microbiota successfully colonize the intestines upon fecal transfer, only the mouse flora can rescue the immune system deficits ${ }^{124}$. Two species of bacteria have been identified separately to be able to mediate this rescue: segmented filamentous bacteria, $\mathrm{SFB},{ }^{124}$ and B. fragilis ${ }^{123}$. Both bacteria have been shown to not only increase $\mathrm{T}$ cell numbers, but also restore inflammatory Th17 T cells and FoxP3+ regulatory $\mathrm{T}$ cells (Tregs), respectively ${ }^{124,126,127}$. In addition, members of the Clostridia genus were shown by another group to be potent inducers of regulatory T cells ${ }^{128,129}$. These are, of course, only a few examples of specific members of the microbiota identified in the past decade to be important for regulating immune function. Recently, integrity of the gut microbiota was also shown to be important for the development 
of microglia, the brain resident macrophages ${ }^{116}$. While the current literature barely scratches the surface, the bidirectional relationship between the gut microbiota and the immune system suddenly seems evident and opens doors to a lot of exciting investigations.

\section{$\underline{\text { Ripples of microbiome effects on the brain }}$}

Through its secreted metabolites and its immunomodulatory factors, the microbiome can affect the function of virtually every organ in the body ${ }^{94,95}$. In terms of interactions between the gut microbiota and the brain, "gut feelings" has been a topic of many review and perspective articles ${ }^{93,130-132}$, unfortunately without measurable supporting data. Early insight about crosstalk between the systems came from patient studies showing high incidence of comorbidities between psychiatric pathology (depression, anxiety, autism) and gastrointestinal pathology (i.e. inflammatory bowel syndrome) ${ }^{131,133-135}$. More recently, small patient cohorts have shown that psychiatric patients (suffering from depression or autism) may actually have distinct microbiota compositions compared to unaffected controls 111,136-139. The main downside of such studies is that the control and experimental groups come from different environments, when household controls would be appropriate for understanding true diseaseassociated differences.

The fact that the gut microbiota is necessary for normal behavior comes from studies investigating the reductionist germ free or antibiotic-treated animals, which have pointed several behavioral and physiological differences compared to their SPF counterparts. Specifically, germ free animals display increased activity and reduced anxiety behaviors ${ }^{111,140-}$ 142, while also showing an exacerbated ACTH and corticosterone release in response to acute stress ${ }^{143,144}$. Germ free mice also show a deficit in social preference compared to SPF raised 
mice ${ }^{145}$. Interestingly, all of these deficits can be recovered by colonization with complete flora before or shortly after weaning time ( 4 weeks), but not once the mice reach adulthood (8 weeks and after) ${ }^{143,145}$. Partial recovery in some behaviors can even be achieved by colonization with a single or a subset of bacteria ${ }^{143}$. In terms of brain physiology, several deficits have been observed in germ free animals, including increased blood brain barrier permeability, altered microglia activity and, more molecularly, decreased levels of BDNF in various brain regions $116,140,143,144,146$. While such studies indicate that the microbiome is necessary to establish normal brain function, they lack the mechanistic insights into what products of the microbiome regulate specific aspects of brain function.

Another line of studies started exploring whether environmental manipulations, such as stress or maternal immune activation, can alter the gut microbiota ${ }^{145,147-152}$. Indeed, it appears that such paradigms do alter the microbiota composition. So far, most of the studies have not explored whether the altered microbiota is associated with behavioral consequences, with a few exceptions. A study by Hsiao and colleagues have shown that maternal immune activation (MIA) induces a microbiota profile which produces elevated levels of 4-ethylphenylsulfate, a previously uninvestigated compound that induces similar behaviors to MIA when injected in naïve mice ${ }^{153}$. Interestingly, the study also showed that administration of probiotic $B$. fragilis, a species not present in the mouse microbiome, can correct the behavioral and metabolic deficits. This result illustrates that an intervention targeting the microbiota may reverse behavioral phenotypes without actually correcting the initial alteration.

In terms of a role for the gut microbiota in modulating depressive behavior, the evidence has been slowly compounding. Patient studies and animal models show that depressed patients and mice exhibiting depressive behavior have different microbiota compositions than controls 
$111,136,137,150,151$. Unfortunately, although these studies show alterations in different bacterial populations, they do not attempt to explain how any of the observed changes may be associated with the behavioral phenotypes. Stronger evidence in favor of the microbiome playing such a role in mediating depressive behavior comes from a recent study employing the "humanized microbiome" technique ${ }^{111}$. Specifically, Zheng and colleagues (2016) transferred fecal contents from depressed and healthy humans into germ free mice and observed that only the mice receiving "depressed microbiota" exhibited despair behavior when compared to the "healthy microbiome" recipients. This is a promising result that suggests that indeed specific microbiota compositions may alter brain function through mechanisms yet uncovered. Indeed, detail is what is particularly missing in the microbiome-brain interactions field. We are yet to understand which component bacteria in the gut microbiome are replaceable, and which bacteria have key roles in maintaining either the microbiota community or aspects of host physiology. Furthermore, we are yet to understand the molecular mechanisms of both bacterial interactions and host-bacteria interactions.

\section{Summary}

So far, I have reviewed the clinical and biological aspects of depression and focused on the gut microbiota as a potential factor influencing the susceptibility to depression. The microbiome is influenced by both host genetics and environmental factors. This barometerlike status puts it in a unique position to be the mediator of previously investigated risk factors, such as diet and inflammation. Current literature indicates that the microbiota composition is 
altered in depressed patients and mouse models. However, our understanding of the functional importance of such differences is very basic, as we lack the knowledge of which specific bacterial changes may be relevant for the observed behavioral phenotypes. Moreover, the mechanisms behind such proposed microbiota-brain interactions are still completely unknown. 


\section{Part II: Involvement of the Gut Microbiota in the Development and Progression of Depressive Behavior}

\section{Hypothesis and aims}

During my thesis work I attempted to answer two questions: what are the specific/ relevant microbiota changes in a chronic stress model for depression and how, mechanistically, can the microbiota affect the nervous system (and behavior)?

Our hypothesis was that chronic stress induces changes in intestinal microbiota composition, which, in turn, alter metabolism or the immune system and feed back on the brain to sustain the development of maladaptive depressive behaviors. We tackled this hypothesis with the following specific aims:

Specific aim \#1: To identify the changes in microbiota composition following chronic stress and what mediates them.

Specific aim \#2: To determine whether the depressive phenotype may be associated with the microbiota composition.

Specific aim \#3: To distinguish contributions of microbiota-affected metabolites and peripheral immune system in mediating the communication between the gut microbiota and the brain. 
Results

\section{Chronic antibiotic treatment induces depressive behavior}

In order to investigate whether general microbiome dysregulation plays a direct role in mediating depressive behavior we first used antibiotic-mediated disruption of the microbiota. While broad-spectrum antibiotic treatment does not fully deplete the microbiota, it does create a severe dysbiosis, making it the second best option to using germ free animals (unavailable to us). Treatment with a broad spectrum antibiotic cocktail for four weeks caused a significant reduction in bacterial load and diversity, as shown by 16s rRNA sequence analysis (Fig. 1a-b). Interestingly, antibiotic-treated mice developed spontaneous depressive behavior, as measured by the forced swim test (Fig. 1c). The assay measures the amount of time an animal struggles to escape an uncomfortable situation, a behavior typically affected in most models of depression and corrected by anti-depressant treatment. To further verify that this was a microbial-dependent effects and rule out a direct effect of antibiotics on the host, mice were treated with the antibiotic cocktail for two weeks and then allowed to recover for 8 weeks. Despite the cessation of antibiotic administration, bacterial load remained reduced (Fig. 1d) and the animals continued to display depressive behavior (Fig. 1e). These findings support our hypothesis that intestinal dysbiosis may contribute to depressive behavior. 

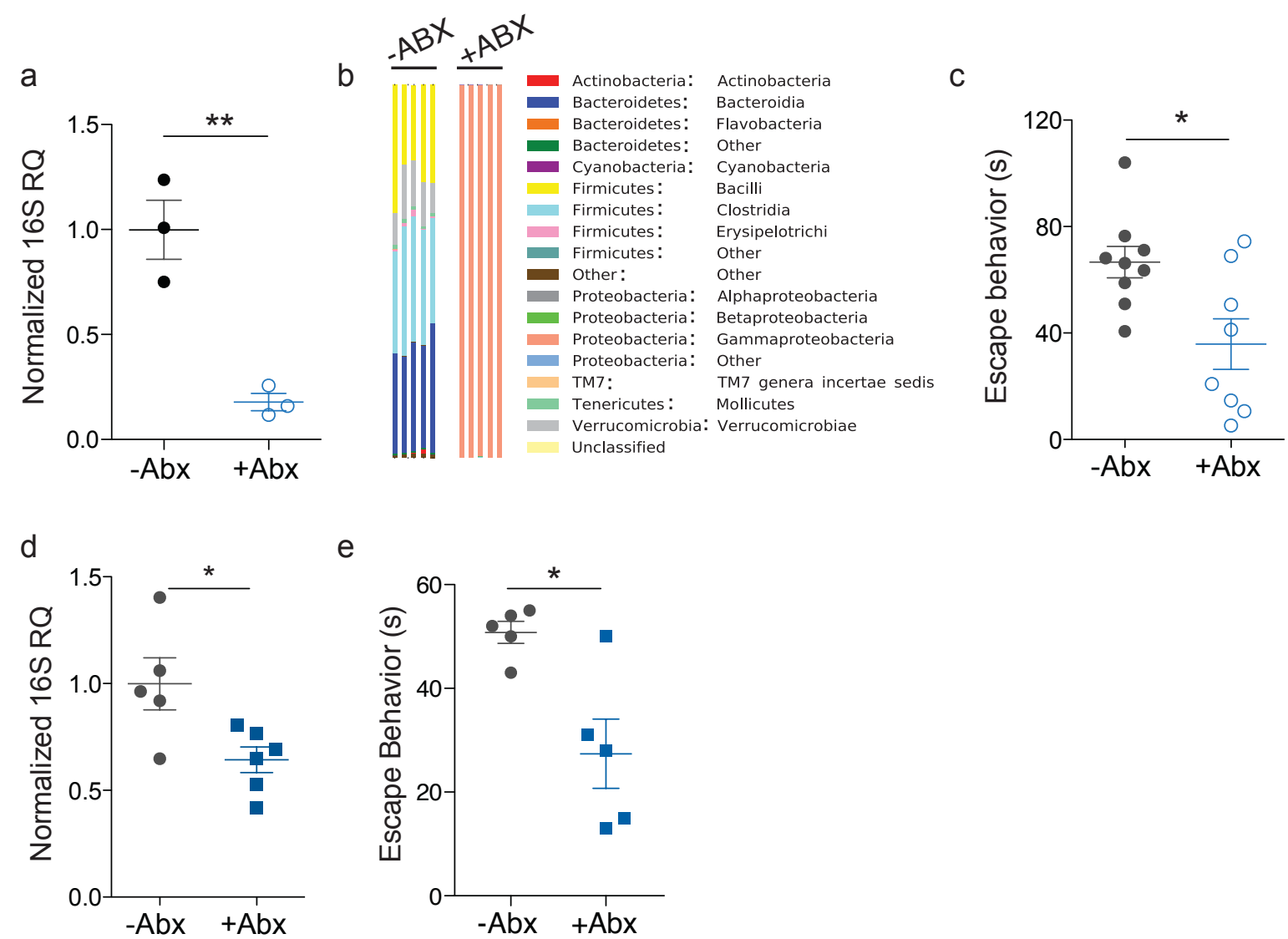

Figure 1. Antibiotic treatment reduces bacterial load and induces depressive behavior. (a) Fecal $16 \mathrm{~S}$ rRNA quantification by semi-quantitative qRT-PCR $\left(n=3\right.$ mice per group; two-tailed t-test, ${ }^{* *} p<0.01$; mean \pm s.e.m). (b) Representative graphs of bacterial class distribution in control and antibiotic treated subjects ( $n=5$ mice per group). (c) Quantification of escape behavior in the forced swim test of control and antibiotic treated mice $(n=9$ mice per group; representative of 3 repeated experiments; two-tailed t-test, ${ }^{* *} p<0.01$; mean \pm s.e.m). (d) Fecal 16S rRNA quantification 8 weeks after cessation of antibiotic treatment, by semi-quantitative qRT-PCR ( $n=5$ control and 6 antibiotic treated mice per group; two-tailed t-test, * $p<0.05$; mean \pm s.e.m). (e) Quantification of escape behavior in the forced swim test 8 weeks after cessation of antibiotic treatment $(n=5$ mice per group; representative of 2 repeated experiments; two-tailed t-test, ${ }^{*} \mathrm{p}<0.05$; mean \pm s.e.m).

\section{Microbiota composition is altered by unpredictable chronic mild stress}

To determine whether chronic stress can directly affect the microbiota, we chose the unpredictable chronic mild stress (UCMS) model of despair behavior ${ }^{7,154}$. Considering the dynamic nature of the intestinal microbiota, we posited that lasting changes must be observed over a prolonged period of time (e.g. weeks-months). The UCMS model seemed particularly 
appropriate due to the length and variety of the stress protocol (Fig. 2a). Consistent with previous reports, this protocol effectively induced despair behavior, as measured by the forced swim (Fig. 2b) ${ }^{14,155,156}$. We verified that the test results were measures of true despair behavior, as the animals showed normal activity and locomotion in the open field test (Fig. 2 c-d).

In order to assess the changes in microbiota composition that occur during chronic stress, we performed 16S rRNA sequencing on genomic DNA isolated from the fecal samples of naïve and stressed mice. The quantity of bacteria in fecal pellets was not affected by stress, as demonstrated by 16S qPCR (Fig. 2e). In terms of microbiota composition, principal coordinate analysis shows distinct clustering between samples from naïve and stressed mice, indicating a significant difference between the groups (Fig. 2f). A more in-depth taxonomic analysis of bacterial types revealed several changes in the microbiota composition (Fig. $2 g$ (i) shows one experimental cohort, Fig. 2g(ii) shows a different experimental cohort; bacterial classes are shown for ease of visualization). In our sequencing runs we observed between 14 and 29 significantly different genera between the naïve and stressed conditions. The variability in the starting microbiota (of naïe mice) and its changes (after stress) is not unexpected, as different shipments of mice, even from the same vendor, can have different microbiota compositions 157,158 . 
a
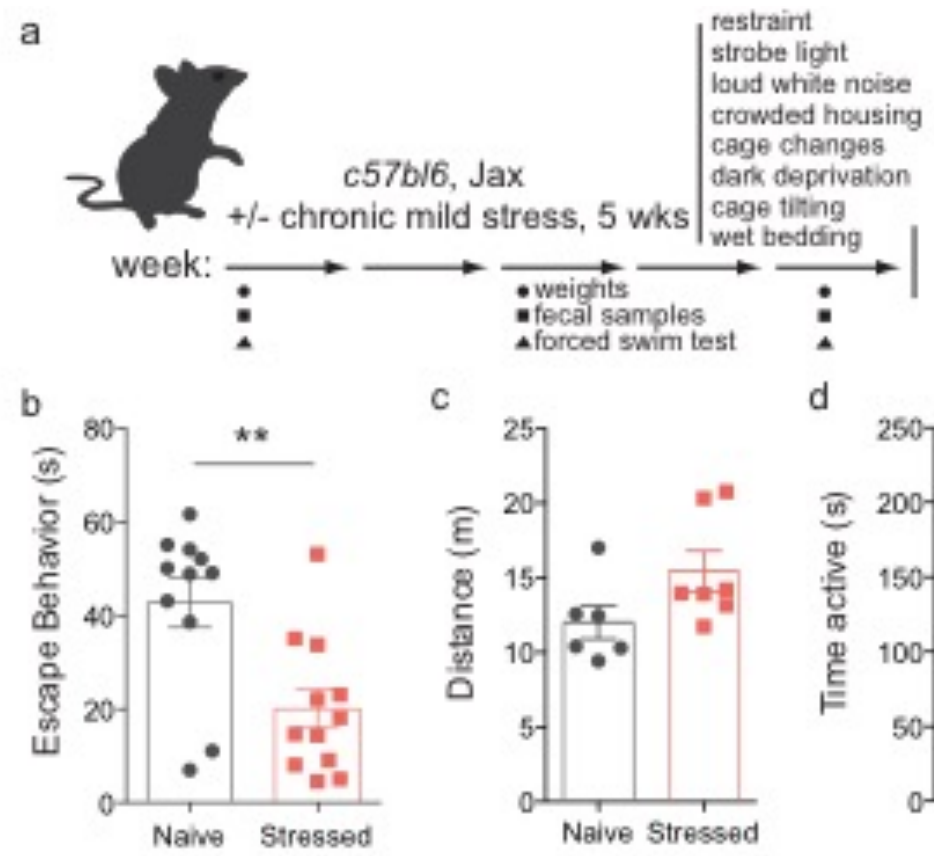

f

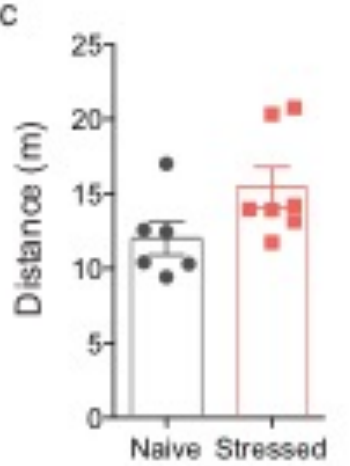

g
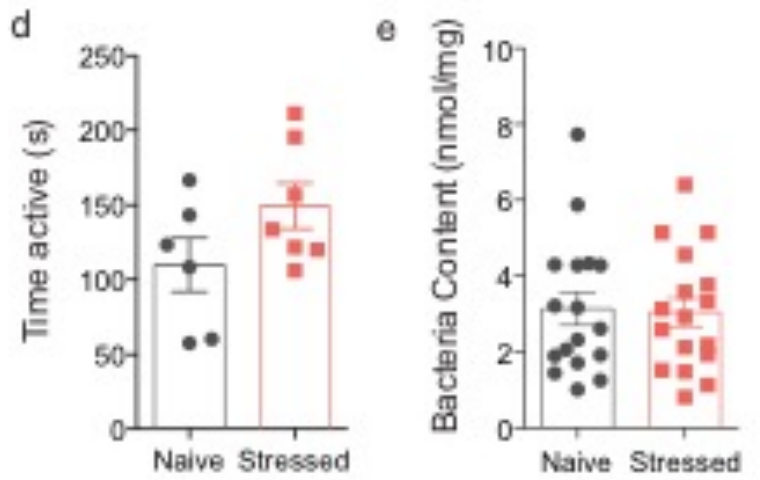

Chronic Mild Stress

Naive Stressed Naive Stressed

(i)

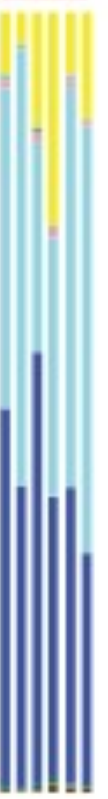

(ii)

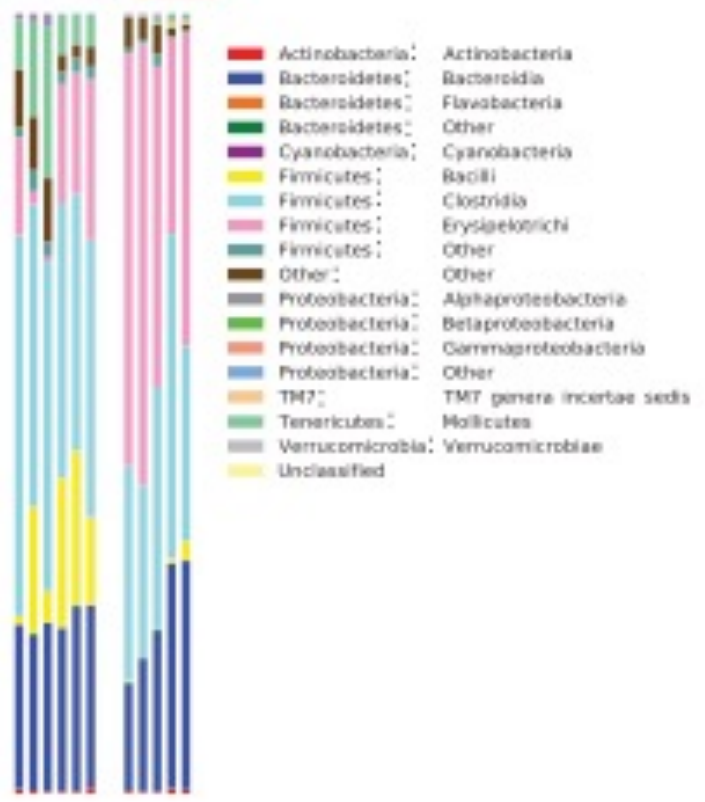

Figure 2. Unpredictable chronic mild stress (UCMS) induces despair behavior and microbiota dysregulation. (a) Experimental design. (b) Quantification of escape behavior in the forced swim test ( $\mathrm{n}=$ 11 naive and 12 stressed; representative of 3 independent experiments; two-tailed t-test, ${ }^{k *} p<0.01$; mean \pm s.e.m). (c) Total distance transversed during the open field test ( $n=6$ naive and 7 stressed). (d) Time spent in the center of the field during a 15 minute test ( $n=6$ naive and 7 stressed). (e) Total bacterial load quantification by qRT-PCR of $16 \mathrm{~S}$ rRNA ( $n=17$ samples per group; two-tailed t-test; mean \pm s.e.m). ( $f$ ) Principal coordinate analysis of microbiome communities in naïve and stressed mice. Analysis based on 2 UCMS experiments ( $n=12$ naïve and 16 stressed; representative of 2 sequencing experiments) (g) Representative graphs of bacterial class distribution in individual subjects show a decrease in bacilli (yellow) ( $\mathrm{n}=5$ naive and 6 stressed; representative of 2 sequencing experiments). 
Overall, the most conserved microbiota change across all independent experiments was a decrease in bacillus class members (both Lactobacillus and Turicibacter) in stressed mice (Fig. 2g). Due to the abundance of literature linking Lactobacilli and behavior and the lack of studies and tools regarding Turicibacter species, we further focused on Lactobacillus as a confident potential player in the despair phenotype.

We verified the net loss of Lactobacillus by qPGR (Fig. 3a) and selective fecal sample cultures (Fig. 3b). These results demonstrate that chronic stress disturbs the microbiota homeostasis, in particular by decreasing the Lactobacillus levels. Correlation analysis returned a positive correlation between the relative Lactobacillus load and the escape behavior displayed by a mouse (Fig. 3c). Our observation was not limited to C57BL/6J, as BALB/cJ (a different inbred strain from Jackson Labs) and C57BL/6N (the same genetic strain from Taconic Farms) mice also show significant correlation between Lactobacillus levels and their escape behavior (Fig. 3d). Interestingly, G57BL/6N mice had very low starting levels of Lactobacillus, which corresponded to low escape behavior even in the absence of stress. Our data are in agreement with recent studies showing associations between lower Lactobacillus levels and stress ${ }^{149,151}$. 

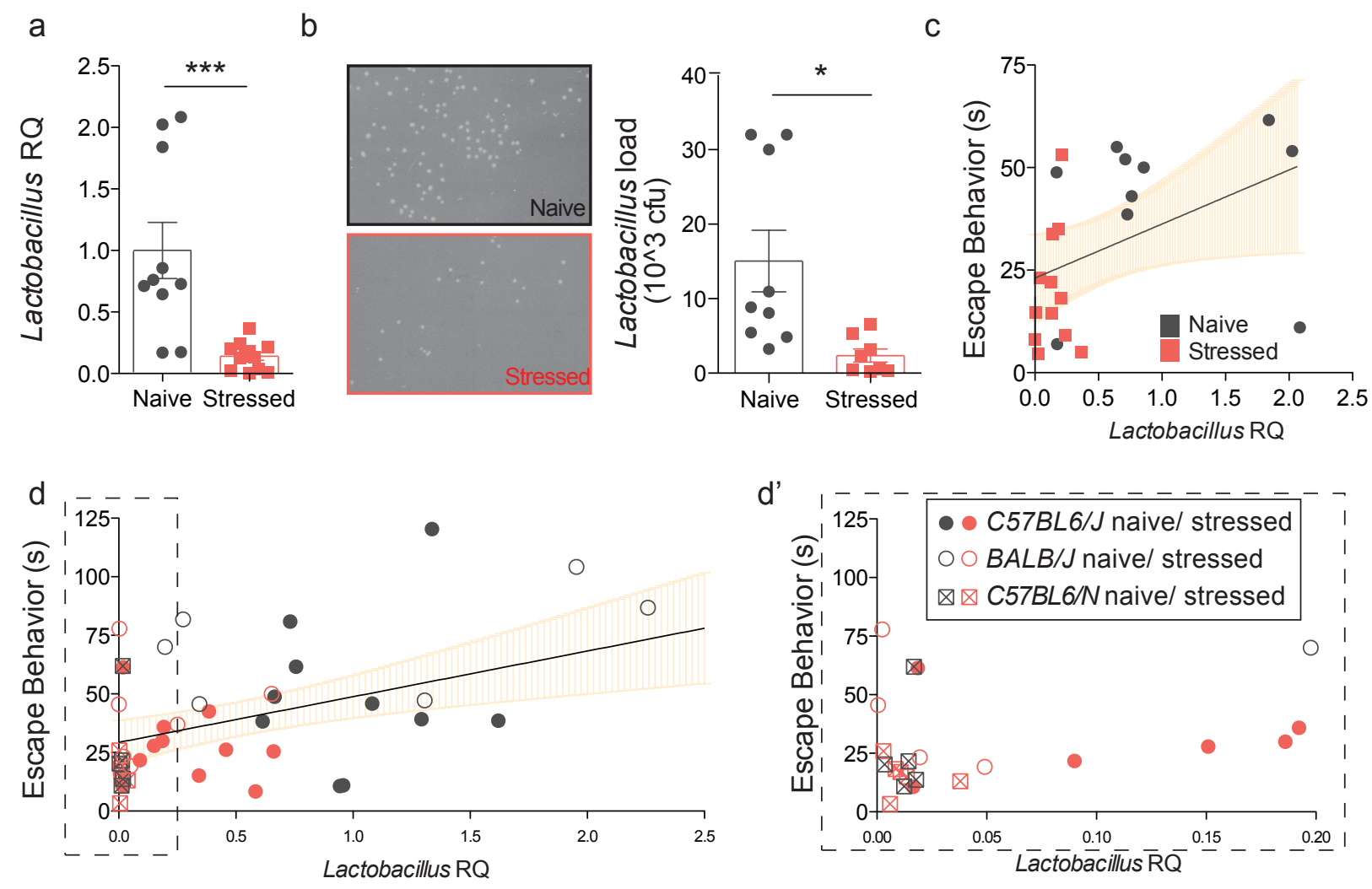

Figure 3. Lactobacillus levels correlate with depressive behavior. (a) Lactobacillus quantification in fecal samples by qRT-PCR, relative to 16S rRNA ( $n=10$ naïve and 11 stressed; representative of 3 independent experiments; two-tailed t-test, ${ }^{* * *} p<0.001$; mean \pm s.e.m). (b) Representative images and quantification of Lactobacillus colonies obtained from fecal samples of naive and stressed mice $(n=9$ naïve and 8 stressed, representative of 2 independent experiments; two-tailed t-test, ${ }^{*} \mathrm{p}<0.05$; mean \pm s.e.m). (c) Correlation analysis between Lactobacillus levels and escape behavior ( $\mathrm{n}=22$ pairs, two-tailed Spearman $r$, ${ }^{* *} p=0.01$, line of best fit with $95 \% \mathrm{Cl}$ ). (d) Correlation analysis between Lactobacillus levels and escape behavior in C57BL6/J (Jax), BALBC/J (Jax), and C57BL6/N (Taconic), naive and stressed $\left(n=45\right.$ pairs, two-tailed Spearman $r,{ }^{* *} p=0.01$, line of best fit with $\left.95 \% \mathrm{Cl}\right)$. (d') Dashed insert with expanded X-axis for better resolution.

\section{Increased intestinal motility, rather than the immune system, may lead to}

\section{the microbiota dysregulation}

To gain insight into potential causes for the changed microbiota composition, we further characterized intestinal immunity and physiology. Surprisingly, intestinal immunity was largely unaltered (Fig. 4a and data not shown), with the exception of a reduction in the Th17 population (Fig. 4b-c). To test whether the Th17 deficit could lead to dysbiosis and depression, 
we sought a mouse model with increased levels of Th17 cells, hypothesizing that it would be protected against depressive behavior. Mice supplied from Taconic Farms are known to have increased type 3 immunity, due to their colonization with segmented filamentous bacteria (SFB) ${ }^{126}$. We compared C57BL/6J mice obtained from Jackson (Jax) to C57BL/6N mice obtained from Taconic (Tac) in their response to UCMS. Tac mice displayed depressive behavior at baseline, which was further exacerbated by UCMS (Fig. 4d). When we compared the Lactobacillus load in the two groups, we observed that Tac mice had significantly reduced levels of the bacteria (Fig. 4e) despite their high Th17 levels. This indicated that the relative abundance of Lactobacilli, rather than the Th17, correlates with the depressive phenotype. To further substantiate this interaction, we attempted to boost Th17 levels in Jackson mice by colonizing them with SFB (Jax+SFB, Fig. 4f). Despite elevated Th17 levels, stressed Jax+SFB mice remained more resilient than their Tac counterparts, while also maintaining higher Lactobacillus levels (Fig. 4g-h). This led us to conclude that it is the Lactobacillus load and not the Th17 levels that correlates with depressive behavior.

To test our hypothesis that the immune system may contribute to the microbiota dysregulation, we subjected SCID mice (lacking adaptive immunity) to UCMS. SCID mice developed UCMS induced depressive behavior to a greater extent than immuno-competent animals (Fig. 5a). To our surprise, though their starting microbiota profile was different from their wildtype counterparts, SCID mice also displayed a significant decrease in Lactobacillus levels after stress (Fig. 5b). 

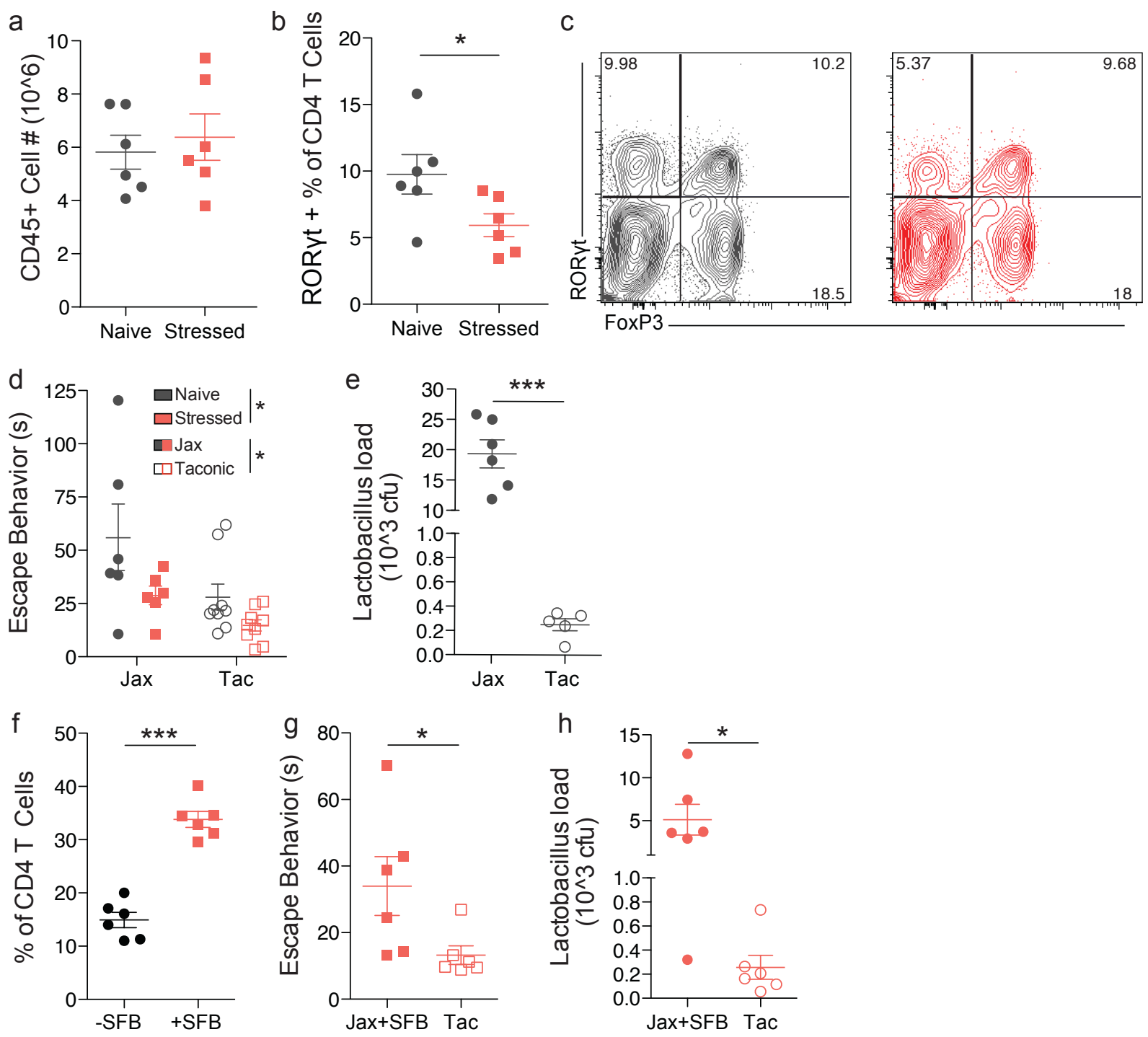

Figure 4. Lactobacillus levels correlate with susceptibility for depressive behavior. (a) Counts of CD45+ cells isolated from the intestine $(n=6$ mice per group; representative of 2 repeated experiments; two-tailed t-test; mean \pm s.e.m). (b) Frequencies of Th17 cells in the lamina propria ( $n=6$ mice per group; representative of 2 repeated experiments; two-tailed t-test, * $p<0.05$; mean \pm s.e.m) (c) Representative flow plots of intestinal CD4+ T cells gated for RORyt and FoxP3 (black - naïve, red - stressed). (d) Quantification of escape behavior in the forced swim test $(n=6$ Jax naïve and stressed, 9 Tac naïve and stressed; 2-way ANOVA followed by Bonferroni post hoc, $\neg$ * $p<0.05$; mean \pm s.e.m). (e) Quantification of selective Lactobacillus cultures from naïve Jax and Tac mice $(n=6$ Jax and 5 Tac; two-tailed t-test, *** $p<0.001$; mean \pm s.e.m). (f) Th17 levels in the small intestine of Jax mice and Jax mice treated with Tac feces (+SFB group) ( $n=6$ mice per group; two-tailed t-test, ${ }^{* * *} p<0.001$; mean \pm s.e.m). (g) Quantification of escape behavior in the forced swim test ( $n=6$ mice per group; two-tailed t-test, ${ }^{*} p<0.05$; mean \pm s.e.m). (h) Quantification of selective Lactobacillus cultures from stressed Jax+SFB and Tac mice ( $n=6$ mice per group; two-tailed t-test, ${ }^{*} p<0.05$; mean \pm s.e.m). 
These data indicate that, while the adaptive immune system may have an important role in mediating resiliency, the chronic stress induced decrease in Lactobacillus is independent of the adaptive immune system. Moreover, supplementing stressed mice with cultured Lactobacillus replenishes their Th17 levels (Fig. 5c-d), indicating that the immune changes observed after stress are likely a product of the altered microbiota.
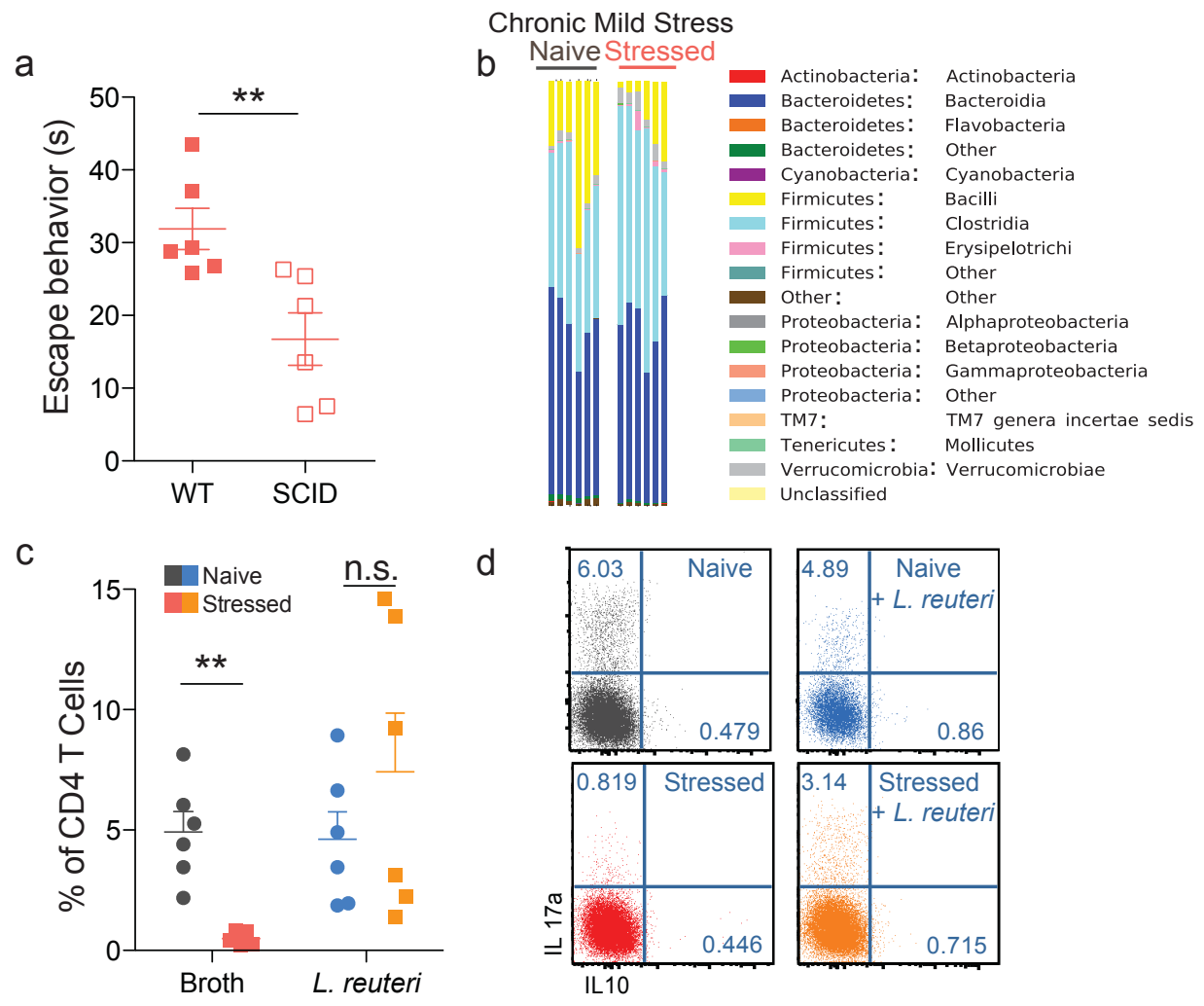

Figure 5. Lactobacillus levels are not dependent on adaptive immunity. (a) Quantification of escape behavior in the forced swim test of stressed wildtype and SCID mice $(n=6$ mice per group; representative of 2 repeated experiments; two-tailed t-test, ${ }^{* *} p<0.01$; mean \pm s.e.m). (b) Representative graphs of bacterial class distribution in individual SCID mice show a decrease in bacilli (yellow) ( $n=5$ naïve and 6 stressed; representative of 2 sequencing experiments). (c) Frequencies of Th17 cells in the lamina propria of naïve and stressed mice treated with either $L$. reuteri or bacteria-free broth $(n=6$ mice per group; two-tailed t-test, ${ }^{* *} p<0.01$; mean \pm s.e.m). (d) Representative flow plots of intestinal CD4+ T cells gated for IL-17 and IL-10.

Given our results, the immune system is the unlikely mediator of the Lactobacillus deficit observed after chronic stress. We therefore turned to our alternative hypothesis, of altered intestinal physiology mediating the microbiota changes. Similarly to previous reports using 
stress models ${ }^{159,160}$, intestinal motility was significantly increased in the stressed animals (Fig.

6a). Furthermore, we observed an increase in the total size and cellular content of the stressed small intestines (Fig. 6b-c). These changes in intestinal physiology in response to stress may underlie the differences in microbiota composition. To more directly test this hypothesis, we treated naïve animals with mineral oil, a non-absorptive lubricant laxative. Increasing intestinal motility in this fashion led to a significant reduction in Lactobacillus levels, as well as in the escape behavior of the treated mice.
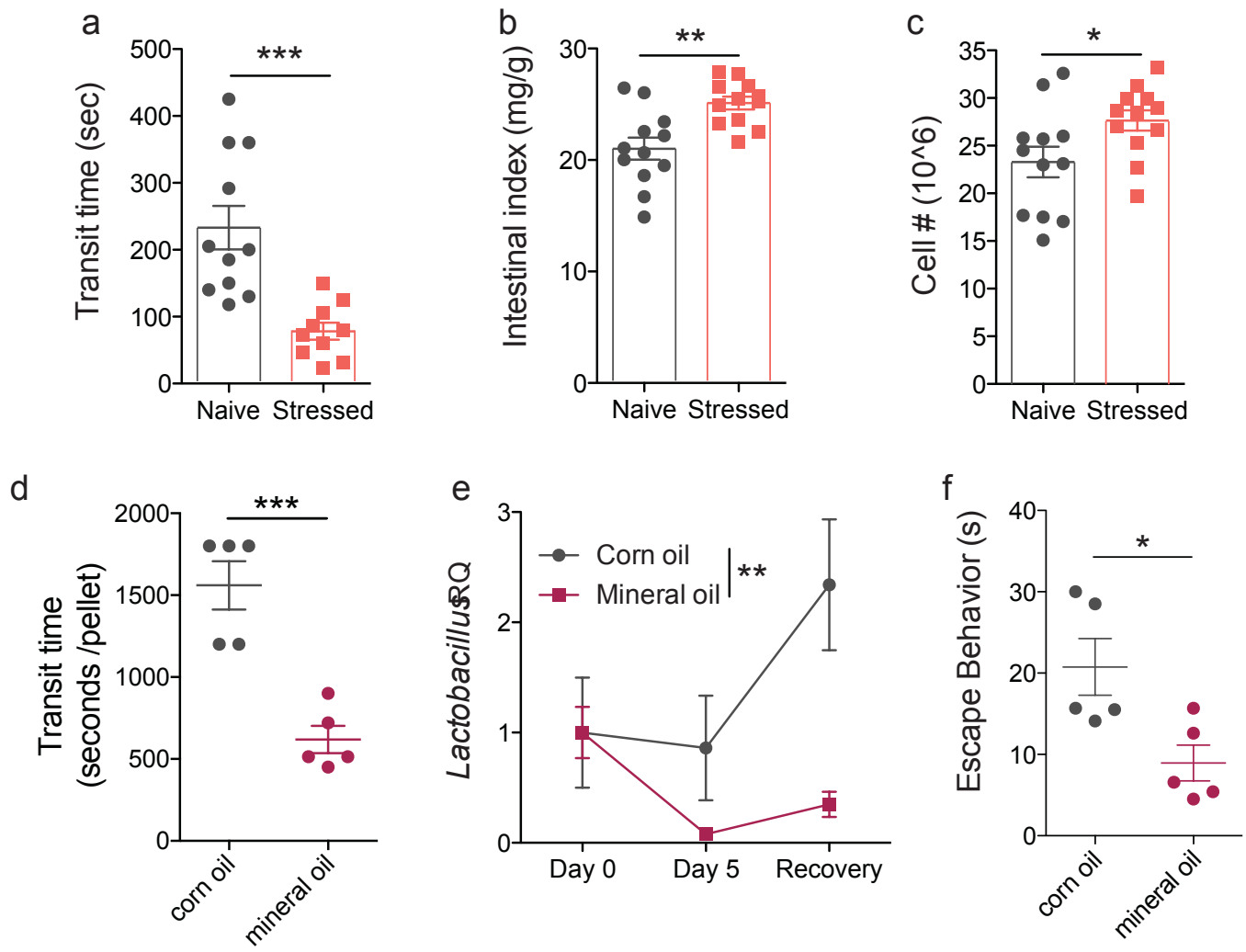

Figure 6. Intestinal motility correlates with depressive behavior and reduced Lactobacillus. (a) Intestinal motility of naive and stressed mice ( $n=12$ mice per group; two-tailed t-test; ${ }^{* *} p<0.001$; mean \pm s.e.m). (b) Small intestine index, relative to body weight $\left(n=12\right.$ mice per group, two-tailed t-test; ${ }^{* *} p<0.01$; mean \pm s.e.m). (c) Total intestinal cell numbers ( $n=12$ mice per group, two-tailed t-test; ${ }^{*} p<0.05$; mean \pm s.e.m).(d) Intestinal motility following mineral oil treatment $\left(n=5\right.$ mice per group; two-tailed t-test; ${ }^{* * *} p<$ 0.001 ; mean \pm s.e.m). (e) Lactobacillus levels are decreased after 5 days of mineral oil treatment, and remain low after 10 days of treatment followed by a 5 -day recovery $(n=5$ mice per group; 2-way ANOVA; ${ }^{* *} p<0.01$; mean \pm s.e.m). (f) Quantification of escape behavior in the forced swim test after 10 days of laxative treatment $\left(n=5\right.$ mice per group; two-tailed t-test, ${ }^{* *} p<0.01$; mean \pm s.e.m). 
These results are preliminary and a more thorough analysis of the microbiota composition, as well as of the behavior would paint a clearer picture. It is likely that Lactobacillus is not the only bacterial genus affected by laxative treatment, though its nutritional requirements do render it especially dependent to a stable environment (161-164, further expanded in the discussion). Nevertheless, these results indicate that fluctuations in intestinal motility can play a role in maintaining the composition of the microbiota.

\section{Treatment with a Lactobacillus species ameliorates despair behavior by restoring kynurenine metabolism}

To assess whether Lactobacillus levels may play a role in mediating despair behaviors, we attempted to replenish the levels of the bacteria and then measure escape behavior. To this end, we subjected mice to the UCMS protocol for three weeks and then supplemented their diet with live cultures of $L$. reuteri, while continuing the stress protocol for additional 3 weeks (Fig. 7a). Lactobacillus reuteri (ATCG 23272) is a species that colonizes several vertebrate hosts, including rodents and humans and has been previously used as a probiotic in mice ${ }^{165}$. This regimen indeed elevated Lactobacillus levels in stressed mice (Fig. 7b). Moreover, in our experimental paradigm, L. reuteri supplementation ameliorated the despair behavior induced by UCMS (Fig. 7c), indicating that Lactobacillus levels may be mediating, at least in part, the behavior.

To get an insight into the mechanism of Lactobacillus-supported resiliency, we performed untargeted metabolomics analysis of serum samples to identify if and how metabolites composition was altered after chronic stress. Principal component analysis showed that the 
metabolic profile of stressed mice is significantly different from that of naïve mice (Fig. $\left.7 d_{i}\right)$.

Treatment with $L$. reuteri converted the metabolic profile of stressed mice to an intermediate profile, suggesting that some of the stress associated metabolic alterations may be a consequence of decreased Lactobacillus levels (Fig. 7d $\mathrm{d}_{\mathrm{ii}}$. While several molecules were significantly different in our analysis (234 out of 4900 spectra), most returned spectra were not confidently matched to known molecules due to limitations in the metabolite library.
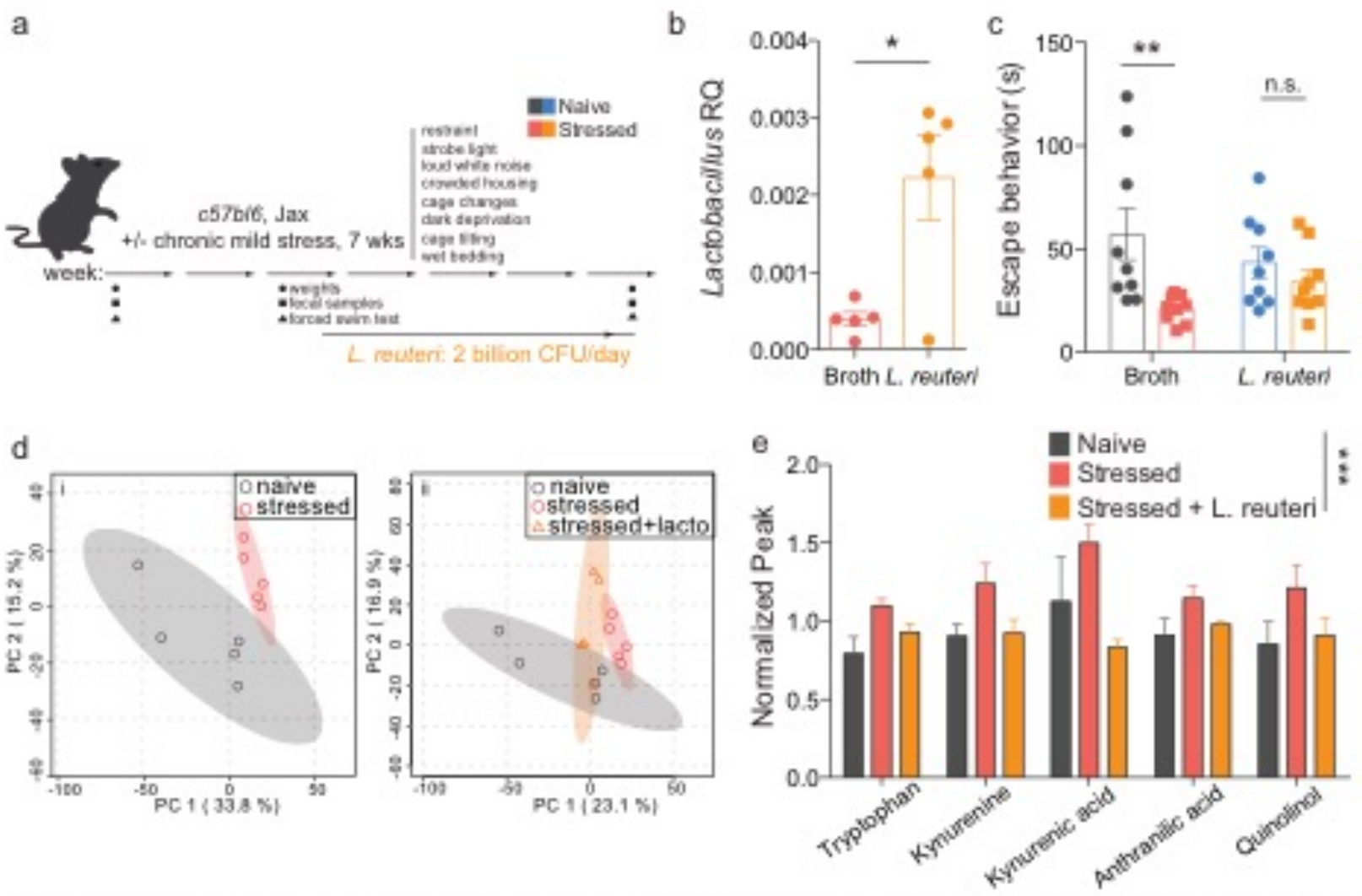

Figure 7. Treatment with probiotic $L$. reuteri ameliorates the escape behavior induced by chronic stress. (a) Experimental design of $L$ reuteri supplementation regimen. (b) qRT-PCR quantification of Lactobacillus levels in fecal samples of $L$. reuteri or broth-control treated mice, relative to 16S rRNA ( $\mathrm{n}=$ 5 ; representative of 2 independent experiments; two-tailed t-test, " $p<0.05$; mean \pm s.e.m). (c) Forced swim test quantification of escape behavior of naïve and stressed mice treated with either $L$. reuteri or bacteria-free broth ( $n=9$ per group; representative of 2 independent experiments; 2-way ANOVA followed by Bonferroni post-hoc, ${ }^{* \prime} p<0.01$; mean \pm s.e.m). (d) Principal component analyses of serum metabolite composition after untargeted metabolomics assay ( $n=5$ mice per group) showing two (i) or three (ii) group comparisons; shaded areas represent $95 \% \mathrm{Cl}$. (e) Normalized MS peaks of tryptophan - kynurenine pathway metabolites in the sera of naive, stressed and $L$. reuteri treated stressed mice ( $n=3-5$ per group; 2-way ANOVA, ${ }^{* * *}$ p $<0.001 ;$ mean \pm s.e.m). 
Of the identified compounds, we mined those increased in stressed animals and normalized by L. reuteri treatment. Among them, metabolites in the tryptophan-kynurenine pathway presented this pattern (Fig. 7e). Evidence of dysregulation of this pathway in depressed patients, as well as its recently described role in the initiation of despair behaviors ${ }^{84}$, have made the tryptophankynurenine pathway particularly compelling as part of the potential mechanism mediating the microbiome effects on behavior. Intriguingly, a recent study ${ }^{166}$ showed that Lactobacillus can directly modulate kynurenine metabolism, by inhibiting the pathway initiating enzyme IDO1 via production of reactive oxygen species (i.e. $\mathrm{H}_{2} \mathrm{O}_{2}$, Fig. 8a). IDO1 is the main enzyme metabolizing L-tryptophan to kynurenine outside of the liver (where TDO is the primary enzyme) ${ }^{167}$.

We verified that cultured $L$. reuteri produced a significant amount of $\mathrm{H}_{2} \mathrm{O}_{2}$ in vitro, when compared to E.coli (Fig. 8b). We next measured peroxide levels in the fecal contents of the stressed mice and discovered that $\mathrm{H}_{2} \mathrm{O}_{2}$ levels were decreased in the stressed mice; in agreement with our hypothesis, therapeutic administration of $L$. reuteri significantly raised the level of $\mathrm{H}_{2} \mathrm{O}_{2}$ in vivo (Fig. 8c). Moreover, we observed a significant correlation with the drop in Lactobacillus levels and the levels of $\mathrm{H}_{2} \mathrm{O}_{2}$ (Fig. 8d). We further verified the kynurenine pathway dysregulation by probing for ido1 mRNA in the intestine, as well as the brain. Our results show increased $i d o 1$ expression in the intestines after stress, while it remained almost undetectable in the brain (Fig. 8e). To further investigate the causative role of kynurenine metabolism in mediating despair behavior we treated naïve mice with L-kynurenine daily (Fig. 8f) and observed a significant reduction in escape behavior at the end of the 4-week protocol (Fig. 8g). 
a

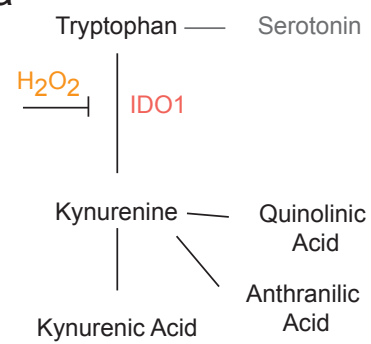

e

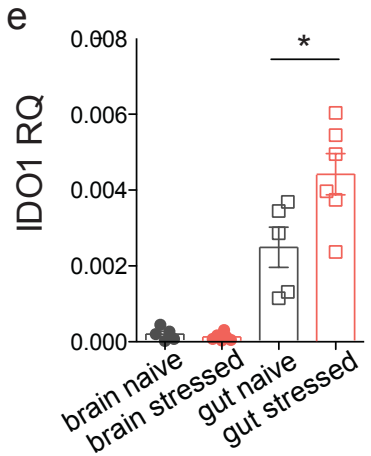

b

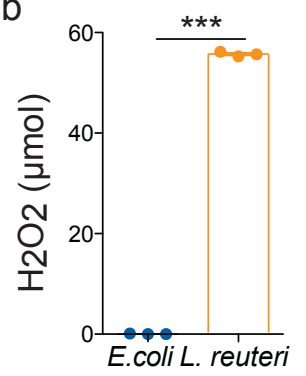

$f$
C

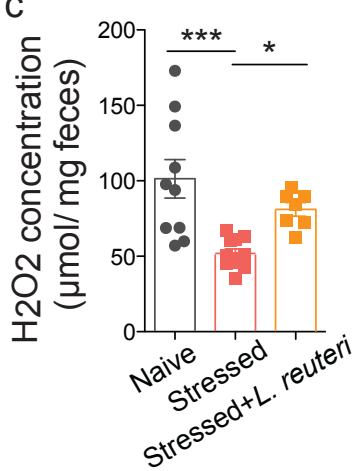

$\mathrm{d}$

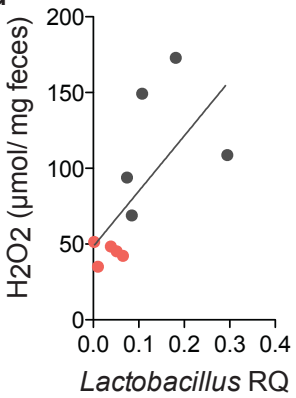

g

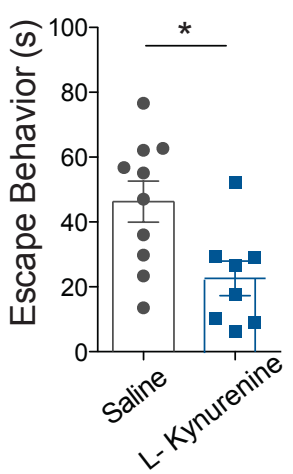

$\mathrm{h}$

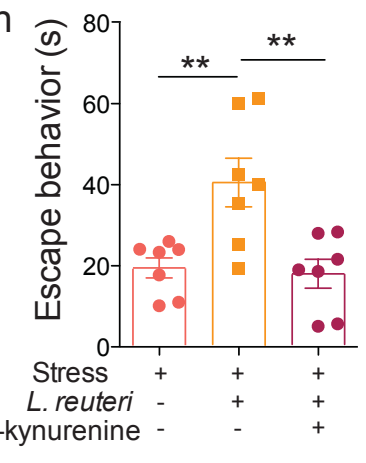

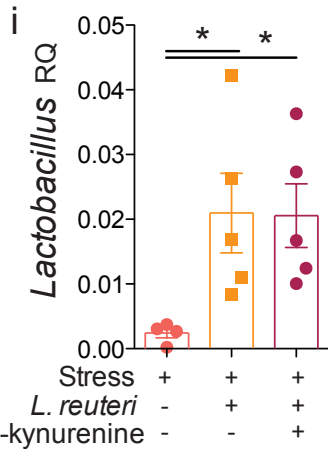

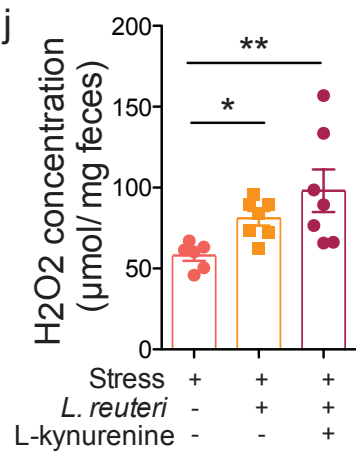

Figure 8. Lactobacillus supplementation has anti-despair activity by moderating kynurenine metabolism. (a) Representation of tryptophan-kynurenine pathway, depicting $\mathrm{H}_{2} \mathrm{O}_{2}$ inhibition of pathway-initiating enzyme IDO1. (b) Production of $\mathrm{H}_{2} \mathrm{O}_{2}$ by $E$. coli and $L$. reuteri $(\mathrm{n}=3$ wells per group; representative of 2 independent experiments; two-tailed t-test, ${ }^{* * *} p<0.001$; mean \pm s.e.m). (c) Fecal $\mathrm{H}_{2} \mathrm{O}_{2}$ levels in naïve, stressed and $L$. reuteri treated stressed mice $(n=7-11$ mice per group; modified one-way ANOVA with Dunn's multiple comparisons, ${ }^{*} p<0.05,{ }^{* * *} p<0.001$; mean \pm s.e.m). (d) Correlation between Lactobacillus and $\mathrm{H}_{2} \mathrm{O}_{2}$ levels in naïve and stressed mice $\left(n=5\right.$ mice per group; Spearman $r$ test, $\left.{ }^{* *} p=0.01\right)$. (e) qRT-PCR quantification of IDO1 expression in the brain and intestines of naïve and stressed mice, relative to GAPDH. ( $n=5$ naïve and 6 stressed, 2-way ANOVA followed by Bonferroni post-hoc, $p<0.05$; mean \pm s.e.m). (f) Experimental design of $L$. reuteri and/ or kynurenine administration. (g) Forced swim test quantification of escape behavior of naïve mice treated with L-kynurenine or saline control $(n=8-10$ mice per group; representative of 2 independent experiments; two-tailed t test, ${ }^{*} p<0.05$; mean \pm s.e.m). (h) Forced swim test quantification of escape behavior of stressed mice treated with either $L$. reuteri alone or $L$. reuteri and L-kynurenine ( $\mathrm{n}=7$ per group; 1-way ANOVA followed by Bonferroni post-hoc, ${ }^{* *} \mathrm{p}<0.01$; mean \pm s.e.m). (i) Quantification of fecal Lactobacillus levels after L. reuteri supplementation and kynurenine treatment $(n=4-5$ animals per group; modified one-way ANOVA with Dunn's multiple comparisons, ${ }^{*} p<0.05$; mean \pm s.e.m) (j) Quantification of fecal $\mathrm{H}_{2} \mathrm{O}_{2}$ levels after L. reuteri supplementation and kynurenine treatment $(n=6-7$ animals per group; modified one-way ANOVA with Dunn's multiple comparisons, ${ }^{*} p<0.05,{ }^{* *} p<0.001$; mean \pm s.e.m). 
Moreover, in order to show that the benefit of Lactobacillus supplementation is by reducing kynurenine levels, we treated stressed mice simultaneously with L.reuteri and L-kynurenine, expecting kynurenine to bypass the benefits of L.reuteri supplementation.

Indeed, while $L$. reuteri alone increased the escape behavior of stressed mice, kynurenine administration abrogated the beneficial effect of L.reuteri (Fig. 8h), even with elevated levels of Lactobacillus and $\mathrm{H}_{2} \mathrm{O}_{2}$ (Fig. 8i-j).

We further attempted to solidify our results by blocking the IDO1-kynurenine pathway. In a preliminary experiment, mice were subjected to the UCMS protocol for 4 weeks, after which they received an inhibitor of IDO1 (1-methyl-tryptophan, 1-Me-Trp), while continued being stressed (Fig. 9a). When tested in the forced swim test, all animals showed reduced escape behavior at the 4-week timepoint. However, after 1-Me-Trp administration, the treatment group showed a trend towards improved escape behavior $(\mathrm{p}=0.17$, Fig. 9b).
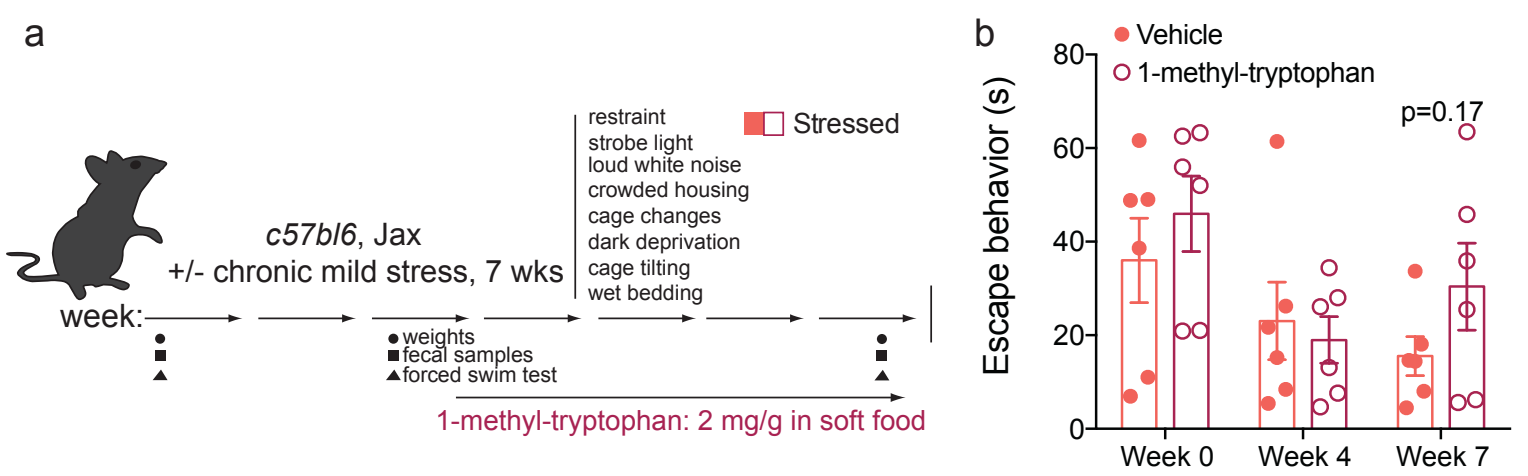

Figure 9. Inhibiting IDO1 may improve depressive behavior. (a) Regimen of administration of IDO1 inhibitor, 2-methyl-tryptophan. (b) Quantification of escape behavior in the forced swim test at the beginning of the stress protocol (week 0 ), after 4 weeks of stress (week 4), and after 3 additional weeks of stress and 1-Me-Trp treatment $(n=6$ mice per group; 2-way ANOVA with Fisher LSD posthoc; mean \pm s.e.m).

Though preliminary, the lack of a larger effect is likely due to the very low dose of 1-Me-Trp used. Further literature search revealed that doses of up to $400 \mathrm{mg} / \mathrm{kg}$ can be administered 
without toxic effects 168,169 . This result complements the rest of data in supporting a role for elevated kynurenine metabolism in mediating depressive behavior.

While so far the data point to a strong association between intestinal Lactobacilli and resilience, it does not show a causal relationship. While selective depletion of Lactobacilli is not feasible with current resources, colonization of germ-free mice with a single or a select set of bacteria has been accepted in the microbiome field. In the absence of a germ-free facility, we attempted to re-colonize antibiotic treated mice with L. reuteri, using a Bacteroides (a genus not changed in stressed animals) species as a control. We first verified selective re-colonization with L. reuteri and B. vulgatus in antibiotic treated mice (Fig. 10a-b). When subjected to the forced swim test, the re-colonized mice showed only mild improvement, irrespective of the bacterial genus they received (Fig. 10c). This result is likely due to the devastating dysbiosis that broadspectrum antibiotic treatment causes, which cannot be restored by a single bacterial species.
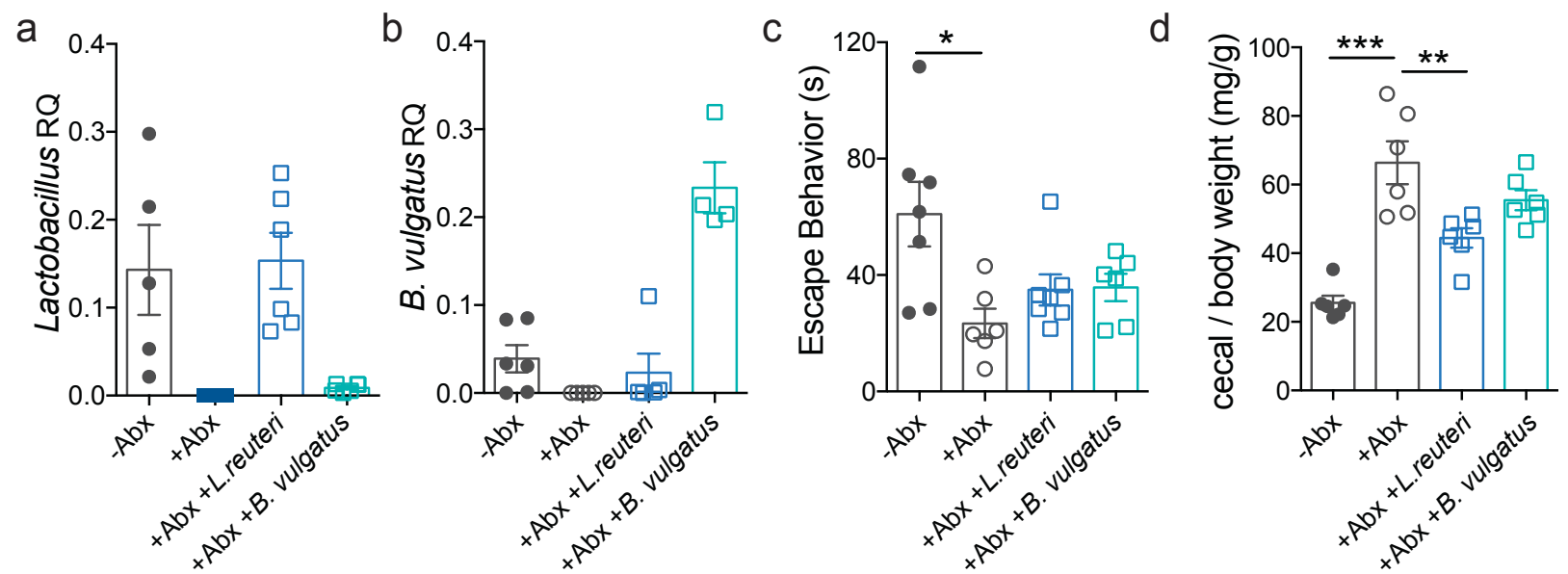

Figure 10. Replenishing Lactobacillus in antibiotic treated mice is not sufficient to recover their behavioral deficits. (a) Lactobacillus levels in the four treatment groups. (b) B. vulgatus levels across the treatment groups. (c) Quantification of escape behavior in the forced swim test after antibiotic treatment and replenishment with $L$. reuteri or $B$. vulgatus $\left(n=5-6\right.$; one-way ANOVA with Tuckey post-hoc, ${ }^{*} p<0.05$; mean \pm s.e.m). (d) Cecal size relative to body weight across treatment groups ( $n=5-6$; one-way ANOVA with Tuckey post-hoc, ${ }^{* *} p<0.01,{ }^{* *} p<0.001$; mean \pm s.e.m). 
Dramatic cecal enlargement is a phenomenon observed in antibiotic-treated mice, as well as germ-free mice, and is a measure of their intestinal health ${ }^{119,170}$. The re-colonized mice remained enlarged, showing only a mild amelioration in their cecal size (Fig. 10d). This data indicate the sustained presence of dysbiosis despite the supply of beneficial bacteria. Considering the downsides of this model, the ideal system for showing causation between Lactobacillus levels and despair behavior, would be early colonization of germ-free mice with a complete, known, flora, containing or lacking Lactobacillus species. Altered Schaedler flora is a model microbiota composed of 8 defined bacterial species (including two Lactobacillus species) corresponding to the most abundant genuses observed in the mouse and human microbiomes 170,171 . Since the flora is introduced by individual cultures of component bacteria, the Lactobacillus species can be left out, thus resulting in a balanced flora missing a specific component. 


\section{Part III: Discussion and Future Directions}

\section{Putting the data in context}

Taken together, our results demonstrate that microbiome homeostasis was robustly altered in animals undergoing UCMS, with a consistent decrease in Lactobacilli. This phenomenon is likely due to the increased intestinal motility associated with stress. Moreover, our data suggest that the production of $\mathrm{H}_{2} \mathrm{O}_{2}$ by Lactobacillus may be protective against the development of despair behavior by direct inhibition of IDO1 expression and decrease in the circulating level of kynurenine, a metabolite associated with depressive behavior (Fig. 11) 84,172-174.

Our results are in agreement with recent literature demonstrating that microbiome composition is modified with acute and chronic stress ${ }^{149-151,175}$. Microbiome dysbiosis is also detected in humans affected by major depressive disorders and the transplantation of the flora from these patients in germ free mice can induce despair behavior ${ }^{111,136,137}$. Beyond describing microbiome fluctuation as a consequence of UCMS, we further demonstrated that levels of Lactobacillus correlate with the susceptibility to and severity of despair behaviors. Indeed, animals exhibiting low (i.e. Taconic C57BL/6N mice) intestinal Lactobacillus levels present with a basal despair phenotype, when compared to animals with higher levels of Lactobacillus (i.e. Jackson C57BL/6J mice). Accordingly, therapeutic administration of Lactobacillus to stressed 
mice, exhibiting reduced levels of the bacteria, was sufficient to improve their despair symptoms.
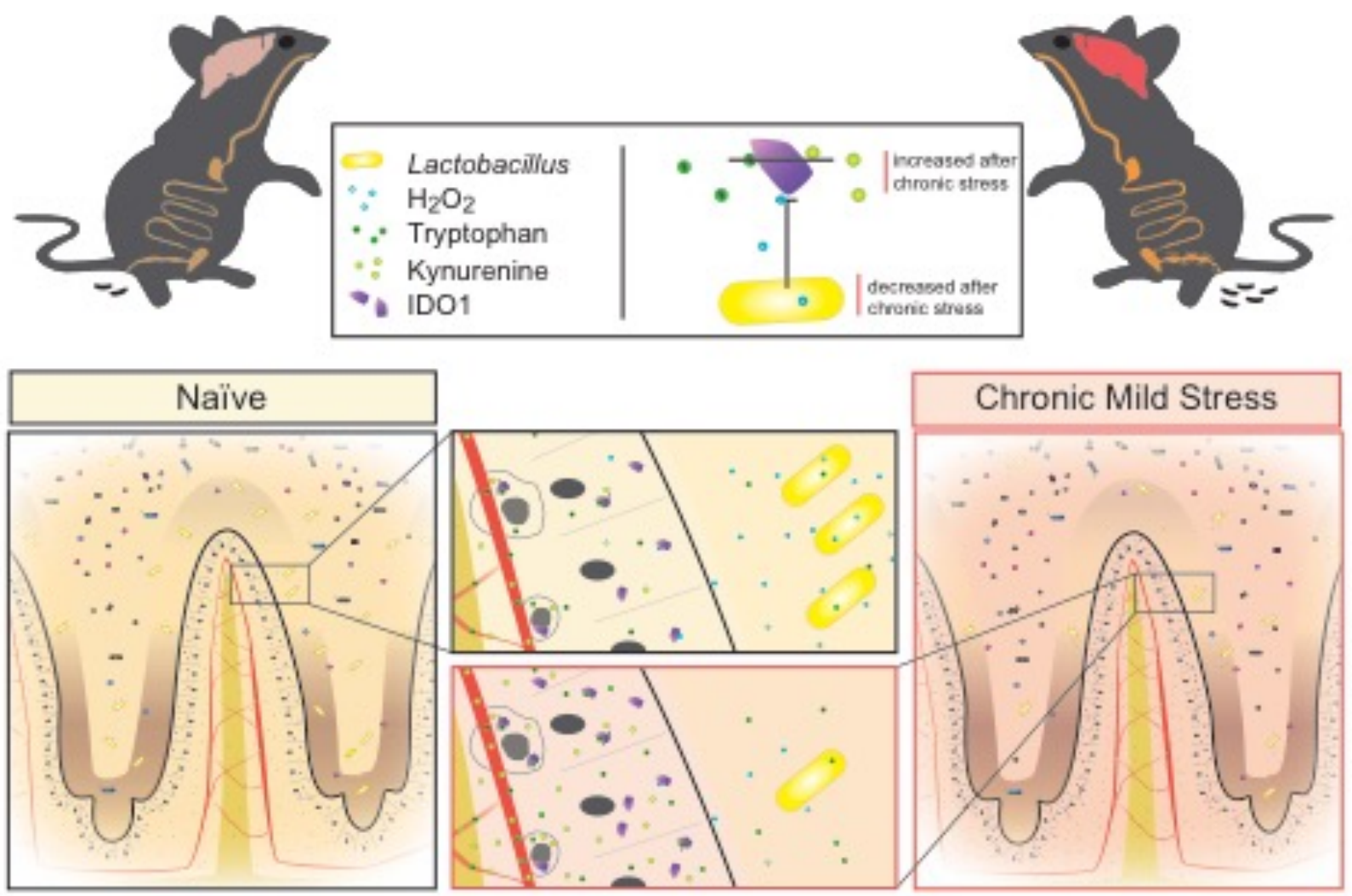

Figure 11. Working model showing downstream effects of microbiota dysbiosis induced by chronic stress. At baseline, sufficient Lactobacillus secrete $\mathrm{H}_{2} \mathrm{O}_{2}$, which may diffuse into the intestinal epithelium and inhibit IDO1 from metabolizing tryptophan to kynurenine. During chronic stress, increased intestinal motility creates a state of dysbiosis, with decreased levels of Lactobacillus. The resulting low levels of peroxide lead to increased IDO1 expression and elevated kynurenine metabolism.

Recently, members of the Lactobacillus genus have been shown to affect a multitude of aspects of human physiology, as they colonize several sites of the body, including the skin, the vagina, and the entirety of the gastrointestinal tract, starting with the oral cavity ${ }^{176}$. Perhaps best studied in the vagina, Lactobacilli protect against infection by producing a diversity of antimicrobial factors, including lactic acid, peroxide, bacteriocins, as well as by resource competition ${ }^{176-178}$. Although in a few contexts increased levels of Lactobacilli are associated with pathology, e.g. dental cavities ${ }^{179}$, the bacteria are largely non-pathogenic or beneficial. From 
dysbioses or probiotic studies, Lactobacilli are associated with protection against infection, improved recovery after enteric infections, decreased colitis pathology, and better cognitive function ${ }^{93,180-182}$.

While Lactobacilli are able to control other microbial communities through secretion of antimicrobial factors, genetic limitations make them more sensitive to environmental conditions. In particular, many Lactobacillus genus members are unable to synthesize amino acids and purines and thus rely on nutrient rich environments and other bacteria for supply of essential building blocks ${ }^{161-164}$. We hypothesized that, in the context of increased intestinal motility such as the one observed in stressed animals, fluctuating availability of nutrients and symbiotic bacteria will impact the renewal of the Lactobacillus niche ${ }^{183}$. Indeed, our results show that directly increasing intestinal transit is sufficient to significantly reduce Lactobacillus levels.

We sought a mechanistic explanation for how the microbiota, and Lactobacilli, in particular, could impact behavioral outputs. By investigating peripheral metabolism, we found that the level of circulating kynurenine is increased after chronic stress, in a manner dependent on Lactobacillus levels. Kynurenine can readily cross the blood-brain barrier to drive depressive behavior, supposedly by disrupting neurotransmitter balance and driving neuroinflammation, within the CNS ${ }^{84,167,174,184-186}$. A recent study by Agudelo et al. ${ }^{84}$ identified this pathway as also being disrupted in stressed mice using the same model of UCMS. The study further shows that peripheral increases in kynurenine translate in dysregulation of downstream pathway metabolites in the brain, possibly altering neurotransmission. Taken together, these new findings point to disruptions in tryptophan-kynurenine metabolism as an important factor in mediating despair behavior. IDO1 is the main enzyme responsible for conversion of tryptophan to kynurenine and its expression and activity are inhibited by reactive oxygen 
species (ROS) ${ }^{166}$. Members of the Lactobacillus family have the capacity to produce high levels of ROS, as a way of fending off other bacteria and maintaining their niche ${ }^{177,178}$. Though difficult to show, it is conceivable that ROS molecules can diffuse from the lumen to signal to the intestinal epithelial cells. In our study, we have shown that decreased levels of ROS in stressed animals correlate with an increase in intestinal ido1 transcripts, thus potentially explaining our observed increase in circulating kynurenine.

Altogether, our results indicate that the microbiome can play a role in the development and symptomatology of depression.

\section{Open-ended questions}

While the data stemming from this project provide new insights into how specific aspects of the gut microbiota can affect behavior, they opened more questions than they answered.

\section{$\underline{1 . \text { Is loss of Lactobacillus sufficient to induce depressive behavior? }}$}

Perhaps the most direct question is that of the causality between the level of intestinal Lactobacillus and depressive behavior. The fact that supplementing reduced levels of Lactobacillus with exogenous bacteria improves behavioral outcome is a good indication that the bacteria has a beneficial role for the host. However, it may not necessarily be representative of the homeostatic state. As previously discussed, since selective depletion of Lactobacillus is not 
currently possible, the ideal experiment would be selective reconstitution of germ-free mice with defined complete flora containing or lacking Lactobacillus components. Another option would be reconstituting germ free mice with fecal contents from naïve and stressed mice. However, previous work shows that, once the new microbiota is established in the new host, its composition changes from the original inoculum 109,111. Therefore, it would be difficult to make conclusions about the original components of the transferred microbiota.

\section{Is Lactobacillus-derived ROS the mechanism behind altered kynurenine $\underline{\text { metabolism? }}$}

Another open-ended question is the connection between Lactobacillus and kynurenine metabolism. Both our and previous data show that Lactobacillus can produce high amounts of ROS $166,176,178$. Previous evidence also shows that that the molecule can inhibit IDO1 activity

166. However, whether it is indeed the ROS produced by Lactobacillus in the lumen, diffusing into the epithelial cell layer to inhibit IDO1, or whether other mechanisms are involved, remains unknown. One approach to understand this process would be to genetically manipulate the Lactobacilli to silence or boost their ROS production. Such an experiment would be technically challenging, given that Lactobacilli evolved several genes involved in ROS secretion $161,162,176,178$. Nevertheless, it would definitely be informative about the importance of Lactobacillus-derived ROS for regulating kynurenine metabolism. Another approach to understanding whether luminal ROS can impact intestinal IDO1 and kynurenine metabolism would involve local delivery or buffering of ROS directly in the lumen, and measuring IDO1 activity in the intestinal tissue. The gastroenterology field has developed several assays to study 
localized responses in the intestines, including creating temporary intestinal loops or catheter implantation ${ }^{187,188}$. This strategy would allow control over luminal ROS levels, as well as their timing, for effective differentiation of their effects. Moreover, the timed control would further allow investigation into the cell types that might respond with modulated IDO1 activity.

\section{How can peripheral kynurenine modulate brain activity?}

More directly relevant to depression etiology is a discussion regarding the role of kynurenine metabolites in the brain. In the absence of inflammation, IDO1 expression in the brain is very low and kynurenine is absorbed from peripheral circulation ${ }^{167,184}$. Evidence shows that kynurenine is trafficked through the large neutral amino acid transporter (LAT1), the same transporter used for its precursor, tryptophan ${ }^{185,189}$. LAT1 may be expressed by all the cell types in the brain, but highest expression has been reported on endothelial cells, as well as astrocytes and microglia ${ }^{190,191}$. The two glial cell types reportedly process kynurenine differently. Astrocytes express KAT2 and metabolize kynurenine to kynurenic acid, whereas microglia express KMO and have quinolinic acid as its product 167,184,191,192. Both molecules have neuromodulatory effects, as they can both alter glutamatergic transmission: kynurenic acid is considered an NMDA receptor antagonist, whereas quinolinic acid is considered an agonist $167,184,193$. These experiments stem from exogenous application of the molecules, showing that kynurenic acid has inhibitory effects, whereas quinolinic acid has excitatory effects $167,184,193,194$. However, nothing is known about the regulation and interaction of the molecules at the synapse. Studies show that, with increased peripheral kynurenine metabolism, both kynurenic and quinolinic acid levels are simultaneously increased in areas of the brain 
84,173,174. Considering their opposing effects, specific regulatory mechanisms must exist to maintain synaptic balance (Fig. 12).
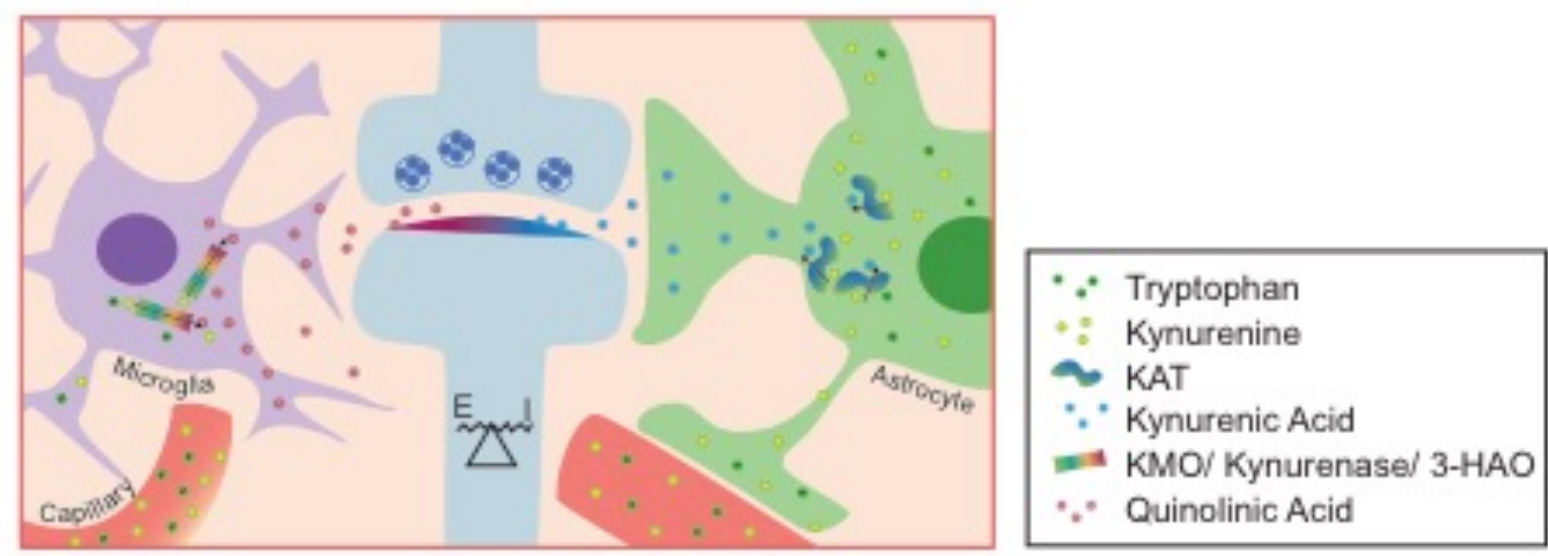

Figure 12. Current understanding of the effects of elevated kynurenine metabolism in the brain. Kynurenine is taken up from the circulation by glial cells. Astrocytes further metabolize kynurenine to kynurenic acid, whereas microglia process it to quinolinic acid. Both compounds are released at the synapse, where they can bind glutamate receptors, with opposite effects. Kynurenic acid has an inhibitory effect, whereas quinolonic acid is excitatory. It is believed that, during homeostasis, the compounds maintain the excitatory/inhibitory balance in the brain. However, increased kynurenine metabolism disrupts this balance, leading to altered neurotransmission and behavioral deficits. (Based on Schwarcz et al. Nat Rev Neurosci 2012)

So far, efforts in investigating the role of kynurenine metabolites in the brain have focused on broad approaches, modulating the amount of kynurenine (and its metabolites), and observing behavioral and cellular effects $84,167,173,174,184,193,194$. This strategy has helped us understand that increased kynurenine levels can induce global changes in neurotransmission, as well as negative behavioral outcomes (impaired cognition, depressive behavior, hyperactivity) $84,173,186,193,195,196$. This is an important step, though just a first step. As discussed in the introduction, attempting to explain the neurobiology of depression with single molecule theories has not been very fruitful. Too much or too little of any molecule can be detrimental 60,82. However, if we focus our further efforts in understanding the cellular effects and regulation of kynurenine metabolites in the brain, we can better comprehend the ways in which peripheral metabolism may affect brain function and contribute to depressive behavior. Important 
outstanding questions involve discerning the role of kynurenine metabolism in different glial cell types and their contribution to synaptic activity. Given the fact that the glial cells express a variety of glutamate receptors ${ }^{197}$, kynurenine metabolites may also impact the function of the glial cells themselves. Moreover, the regulation and timing of release, as well as clearance from the extracellular environment of these molecules remains to be investigated. Are kynurenic and quinolinic acid released simultaneously at the same synapse, or is their timing carefully controlled? Furthermore, if they both indeed bind the same glutamate receptors (as it is assumed), which molecule wins the competition? Finally, more thorough analysis indicates that kynurenine metabolism is not upregulated indiscriminately throughout the brain, but may be limited to discrete areas ${ }^{84}$. Understanding how this process is regulated (is it dependent on neuronal activity?) will also further our knowledge of how kynurenine metabolism may lead to depressive behavior.

\section{What is the role of the immune system in maintaining resilience?}

While evaluating the contribution of the immune system to the microbiota changes during chronic stress, we observed that mice lacking their adaptive immune system (SCID mice) develop an exacerbated depressive phenotype. Giving the vast literature showing that activation of the immune system (both adaptive and innate) is detrimental ${ }^{77-81}$, this is an intriguing finding. Nevertheless, full immune competence has also been shown to be necessary for other behaviors, such as learning and memory ${ }^{198-202}$. Despite these previous studies, we are still lacking the understanding of how the adaptive immune system supports brain activity. One of the studies showed that immunodeficient mice have decreased adult neurogenesis ${ }^{200,201}$, a 
process often associated with depressive behavior due to its recovery by antidepressant treatment ${ }^{203,204}$. Though disrupted adult neurogenesis is often cited as accompanying depressive behavior in animal models $14,89,203,204$, it is unclear whether or how this process may contribute to the depressive behavior. Most adult generated neurons are inhibitory interneurons ${ }^{205,206}$. Given the increased activation of circuits in the hippocampus, as well as the habenula and the VTA, one possibility is that adult neurogenesis is necessary to maintain a stable tone within brain circuitry. Of course, it is also possible that the increased activation of hippocampal circuits inhibits the integration of inhibitory neurons.

Another study investigating cognition in immunodeficient mice showed that the phenotype of the immune cells in the meningeal spaces is associated with the learning outcome of the animals ${ }^{198,207}$. Specifically, mice displaying a meningeal pro-inflammatory skew (such as the SCID mice, IL4 deficient mice) were poor learners compared to the mice that could skew their meningeal repertoire to a pro-homeostatic phenotype (immunocompetent mice). Whether the inflammatory phenotype in the meninges is sufficient to drive cytokine levels high enough to change synaptic activity as seen during systemic inflammation is unknown. Nevertheless, preliminary data shows that SCID mice have higher circulating levels of lipopolysaccharide (LPS), as well as decreased levels of meningeal myeloid cells (Fig. 13a-b). LPS is considered a potent activator of the immune system, usually leading to increased proinflammatory cytokines and blood brain barrier (BBB) permeability ${ }^{81,208}$. The effects of tonically elevated LPS levels, however, are unknown. It is likely that sustained exposure to LPS mediates a wide array of effects, such as a more reactive microglia phenotype, as seen in brain areas with a more permeable $\mathrm{BBB}{ }^{116,209}$. Interestingly, this hypothesis involves the co-existence 
of immunodeficiency and inflammation, two phenomena that are not usually associated together.
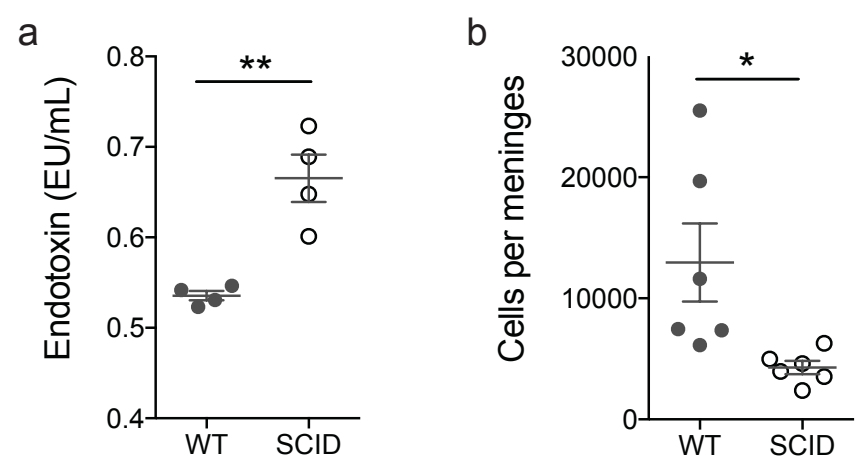

Figure 13. SCID mice show signs of simultaneous inflammation and immunosupression. (a) Serum endotoxin levels ( $n=4$ mice per group; two-tailed t-test, ${ }^{* *} p<0.01$; mean \pm s.e.m). (b) Counts of myeloid cells isolated from the meninges ( $n=6$ mice per group; two-tailed t-test, ${ }^{*} p<0.05$; mean \pm s.e.m).

Undoubtedly, these are just few of the hypotheses to be considered regarding the role of the immune system in maintaining brain function. Following up on this topic will bring important advances about the balance between beneficial and detrimental immune responses for depressive behavior, as well as other aspects of host physiology.

\section{Can gut microbiota dysregulation mediate human depression?}

Whether the activity of a system so far removed from the brain as the gut microbiota can alter its activity to the point of causing a disorder as complex as depression is an argument to consider. In the introduction, I described how the causes of depressive disorder are still unclear. Associated risk factors range from intrinsic (genetics) to environmental ones (stress). Moreover, many diseases, such as autoimmune multiple sclerosis, are hallmarked by depressive behavior 77,78. In the case of multiple sclerosis, for instance, we are still uncertain of what brain changes 
lead to depressive behavior, specifically. However, it is believed that widespread neuroinflammation affects neuronal activity leading to a plethora of altered behaviors, including cognition, resilience and exploration ${ }^{79,81}$. In this context, it is possible that microbiota dysregulation, through widespread metabolism dysregulation, can also alter neuronal activity and lead to behavioral deficits.

Can this happen in humans? The wide array of depression symptoms and co-morbidities indicates that multiple underlying causes might lead to depressive behavior. Therefore, it is conceivable that a subset of patients might suffer from dysbiosis, (perhaps with low levels of Lactobacillus) which underlies or contributes to their depression symptoms. The scenario presented by our experimental model is definitely reductionist and much simpler that any scenarios involving human biology. Nevertheless, in favor of our hypothesis, human studies show high incidence of depression in patients of chronic intestinal disease 210-212.

In this study, we identified Lactobacillus as a genus of bacteria important for mediating resilience, potentially by maintaining kynurenine metabolism. To my knowledge, this is the first study associating a specific type of bacteria with depressive behavior. The finding is undoubtedly dependent on our experimental procedure. However, this is likely just one of many bacteria, which, when dysregulated, may mediate depressive behavior ${ }^{111}$. These remain to be discovered. 


\section{New avenues: the role of kynurenine metabolism in multiple sclerosis associated depression}

Much of our current understanding about the role of kynurenine metabolism in depression comes from models of inflammation ${ }^{173,213,214}$. Of particular interest is multiple sclerosis (MS), an autoimmune disease hallmarked by inflammatory bouts and CNS demyelination, leading to sensory and motor dysfunction. As already mentioned, many MS patients additionally suffer from psychiatric symptoms, such as depression and anxiety ${ }^{215-217}$. Therefore, a potential new avenue stemming from my project would be the investigation of the role of kynurenine metabolism in depression during multiple sclerosis.

Extensive research has been dedicated to discovering pathways and molecules acting on the immune system to limit demyelination during MS attacks ${ }^{218,219}$. Interestingly, one of such promising targets is the tryptophan-kynurenine pathway ${ }^{216,220}$. During inflammation, IDO1, the enzyme that metabolizes tryptophan to kynurenine, is upregulated by various immune cells 173,221-223. Kynurenine and kynurenine associated metabolites have a robust anti-inflammatory activity and are protective in the mouse model of MS: experimental autoimmune encephalitis (EAE) 220,224 . Furthermore, mice lacking IDO1 present with a more severe clinical form of the disease ${ }^{222}$. It is also suspected that IFN $\beta$, one of the most widely used MS treatments, could be acting by inducing IDO1 expression in immune cells ${ }^{216,224}$.

While IDO1 expression and the presence of kynurenine metabolites is beneficial on the immune system in the context of MS 220,222,224, the same player has now been shown to have negative impacts on mental health $84,173,186,193,195,196$. Teasing out the cellular biology of kynurenine metabolism may be particularly impactful for managing depressive behavior in 
MS/EAE. While kynurenine metabolism is increased during MS/EAE, whether this increase is resulting only from IDO activity in immune cells, or whether cells in the brain also increase IDO expression in response to inflammation, is currently unknown. Answering this question could help targeting kynurenine metabolism to harness its beneficial effects in the immune system, while limiting its harmful effects for the CNS.

Recently, loss of oligodendrocyte progenitor cells (OPCs) was shown to initiate depressive behavior in animal models ${ }^{225}$. This subset of glial cells, precursors to myelinating oligodendrocytes, fluctuates in number during demyelinating inflammation, particularly at lesion sites ${ }^{226,227}$. Though kynurenine metabolism in oligodendrocyte lineage cells has not been investigated, it is plausible that kynurenine and/or its metabolites are toxic to these cells and impair their neuro-supportive function.

Therefore, one possible hypothesis is that, in the context of EAE inflammation, IDO/ kynurenine metabolism dampens inflammation at the expense detrimental effects on brain function leading to depressive-like behavior. Specifically, we hypothesize that kynurenine metabolites mediate this behavior in part by impairing the function of oligodendrocyte lineage cells. To address this hypothesis, we will first resolve the contribution of the immune system versus the brain (and other organs) to IDO1/ kynurenine metabolism increase during EAE inflammation and how each contributes to depressive behavior.

To this end, we will generate bone marrow chimeras, in which the host and the immune system will be either wildtype or IDO1 knockout. Upon EAE induction, we will evaluate the animals for disease progression (clinical score, severity of inflammatory response, depressive phenotype). We will also interrogate IDO1 and kynurenine levels in different compartments and cells types associated with autoimmune inflammation and/or kynurenine metabolism. (i.e. 
peripheral immune, central immune, neural and glia cells, intestinal and liver cells). The results should indicate whether the source of IDO1/ kynurenine is solely from immune cells, or whether other cells also upregulate it in response to inflammation. For instance, a chimera with IDO1 knockout immune system, and a wildtype host should present higher clinical scores, but no depressive behavior. In this scenario, reducing kynurenine uptake in the GNS in a wildtype animal (such as by pharmaceutically blocking the LAT1 transporter) could prevent or alleviate depressive behavior, while maintaining its benefits on the immune system.

For the second part of the hypothesis, we will seek to understand the role and effects of IDO1/kynurenine metabolism in oligodendrocyte lineage cells and how it relates to depressive behavior during EAE. To this end, we will use the NG2-eGFP reporter to sort cells at different stages of the oligodendrocyte lineage and screen them for enzymes in the IDO1/kynurenine pathway. We will also use in vitro cultures to determine the effects of kynurenine and its downstream metabolites (kynurenic acid, quinolinic acid, anthranilic acid), on OPC and oligodendrocyte fuction, measuring outcomes such as survival, proliferation, differentiation, myelin production, etc. Such experiments could yield particularly important insights into oligodendroglia function during EAE, as both OPCs and oligodendrocytes express various glutamate receptors, which can bind both kynurenic and quinolinic acid. Recently, activation of NMDA receptors on oligodendrocytes were shown to be important for providing metabolic support to active axons, and deleting these receptors on oligodendrocytes can lead to impaired axonal transmission. It is plausible, therefore, that accumulation of kynurenic acid, an NMDA receptor antagonist, also impairs oligodendrocyte function in providing metabolic support. Finally, following our preliminary experiments regarding kynurenine metabolism in oligodendrocyte lineage cells, we will attempt to block the detrimental effects of kynurenine 
during EAE in vivo by specifically knocking out the identified enzymes using viral delivery of floxed constructs in animals expressing NG2-driven cre protein.

Altogether, these experiments will not only differentiate the source of kynurenine during neuroinflammatory episodes, but will also explore a completely new area of study, the role of kynurenine metabolism in oligodendrocyte lineage cell function. 


\section{Materials and Methods}

Animals. Male C57BL6, BALB/c and SCID (C57BL6) mice (8 weeks old) were purchased from Jackson. For the experiments comparing Jackson and Taconic mice, age-matched C57BL6 animals were purchased from each vendor. The mice were maintained on a 12 hours light/dark cycle with lights on at 7am. All procedures were conducted in accordance to the University of Virginia IACUG guidelines. Chronic stress was started after at least 1 week of acclimation. All behavioral interventions were performed between 4pm and 7pm and takedowns were performed between 11 am and 2pm. Sample size was selected to be similar to previously reported behavioral experiments. Animals were housed 2-3 per cage and cages were randomly assigned to control or experimental groups. Investigators were not blinded to the group allocation. Experiments were conducted in such a way as to make sure that all experimental groups were exposed to the same environments. Animals were excluded from the experiments if they developed illnesses (e.g. dermatitis) that might affect the outcome of the results. Certain measurements are occasionally not available for each mouse enrolled in the study due to technical issues (e.g. fecal sample not provided, sample loss).

Antibiotic treatment. Mice were administered a broad-spectrum antibiotic mix as previously described ${ }^{119}$. The cocktail consisted of ampicillin, kanamycin, metronidazole, and vancomycin, each at $1 \mathrm{~g} / \mathrm{L}$, in drinking water. Fresh antibiotic water was replenished every 2- 
3 days to prevent precipitation. The weights of the animals, as well as their food and water intake were monitored daily for the initial week of treatment, and then every 3 days.

Ghronic mild stress protocol. Mice were subjected daily to an acute stressor (1-2 hours: restraint, loud white noise, crowded housing, strobe light) and an overnight stressor (12-24 hours: $45^{\circ}$ cage tilting, repeated cage changes, wet bedding, dark deprivation) presented in a randomized fashion as described in the literature ${ }^{7}$. For restraint stress, mice were placed in clean $50 \mathrm{~mL}$ conical tubes with pierced holes for ventilation for 1 hour. For crowded housing stress, mice were placed on top of the cage wire for 2 hours, after which their cage was changed. For wet bedding stress, $200 \mathrm{~mL}$ of water was added to the bedding of a clean cage. All procedures used autoclaved, sterile materials (bedding, water) in order to prevent contamination. Food and water intake were monitored in initial experiments and no changes were observed in the stressed group. The weights of the animals were monitored weekly. Sequencing results showed no new OTUs in the stressed microbiota, indicating the lack of contamination during the stress protocol.

Behavioral assessment. Behavioral testing was conducted between 4 and 6pm, with the animals being brought in the testing room 30-60 minutes for acclimatization. Despair behavior was assessed at time points of interest using the forced swim test, as described in the literature 228. The last 4 minutes (out of 6 minutes total test) were scored for escape behavior, defined as active swimming, with all four limbs and tail moving. The test was conducted using autoclaved water and disinfected containers. General mouse activity and locomotion was assessed using the open field test. The mice were placed in the corner of a square arena $(40 \mathrm{~cm} \mathrm{x} 40 \mathrm{~cm})$ and 
allowed to move and explore freely for 15 minutes. Their activity was analyzed live using the EthoVision tracking system.

DNA isolation. Whole genomic DNA was isolated via phenol-chloroform extraction. Briefly, a fecal pellet was placed in a $2 \mathrm{~mL}$ tube containing $200 \mu \mathrm{L}$ silica-zirconia beads $(0.1 \mathrm{~mm})$. The tube was filled with $750 \mu \mathrm{L}$ extraction buffer, $200 \mu \mathrm{L} \mathrm{20 \%}$ SDS and $750 \mu \mathrm{L}$ phenolchloroform-isoamyl alcohol (25:24:1). After disruption, the aqueous phase was separated by centrifugation and cleaned up with two washes of chloroform-isoamyl alcohol (24:1). The DNA was precipitated and resuspended in $10 \mathrm{mM}$ Tris solution for use in other assays.

Fecal microbiota assessment. For $16 \mathrm{~S}$ rRNA sequencing, the V3-V4 region of the $16 \mathrm{~S}$ rRNA gene was amplified for 25 cycles using specific primers with adapter overhangs as per the Illumina library preparation guide. Following purification of the PCR products, individual indexes were added to the amplicons by PCR. The amplicons were purified, pooled in equal quantities, and then sequenced on the Illumina MiSeq platform. Reads with an average quality score below 25 (from any 10-bp window) or a mismatched barcode were removed. Paired-end reads were then merged using the software FLASH ${ }^{229}$. Merged reads were analyzed using the QIIME pipeline with default parameters to remove chimeric, pick 97\%-identity OTUs and assign taxonomy ${ }^{230}$.

Selective culture of lactobacilli. Fecal pellets were resuspended in $1 \mathrm{~mL}$ of deMan, Rogosa and Sharpe (MRS) broth supplemented with sodium azide $(0.02 \% \mathrm{w} / \mathrm{v})$ to select Lactobacillus 
231. After brief decanting of insoluble fecal material, samples were further diluted 1:1000 in MRS/az and $50 \mu \mathrm{L}$ of this dilution were spread on MRS/azide agar plates and grown overnight at $37^{\circ} \mathrm{C}$. The plates were then imaged with a Bio-Rad gel imager and colonies were counted using the "particle counter" plugin in ImageJ.

Bacteria preparation for feeding experiments. For lactobacillus supplementation experiments, L. reuteri was obtained from ATCG (23272) and cultured aerobically according to manufacturer instructions. Briefly, the lyophilized stock was resuspended in $1 \mathrm{~mL}$ of freshly prepared MRS broth and expanded in a starter culture $(5 \mathrm{~mL})$. The culture was stopped while in the logarithmic growth phase, and the bacteria were washed and resuspended in fresh MRS broth. Glycerol aliquots (1mL, 30\% final glycerol concentration) were prepared and stored at -80C. Fresh $L$. reuteri was cultured for oral supplementation by resuspending one aliquot in 250 $\mathrm{mL}$ of MRS broth and growing until optical density reached roughly 0.7. Lactobacillussupplemented food was prepared by pulverizing radiated food pellets in a blender and kneading with fresh $L$. reuteri (2 billion CFU/mouse/day) and water, or with MRS culture broth and water for the control. The animals received fresh food prepared daily and one $L$. reuteri culture was used for 7-10 days. Fresh cultures of Bacteroides vulgatus (ATCG 8482) were used as a control for mono-reconstituted antibiotic treated mice. The bacteria were procured as a minipack of glycerol stocks and stored at -80C. As needed, stocks were thawed and suspended in Reinforced Clostridia Media preloaded in anaerobic bottles (BD 215192). The bottles were incubated in an airtight container equipped with two GasPak EZ anaerobe pouches (BD 260683). An appropriate volume of bacterial culture (for 2 billion CFU/mouse/day) was extracted using a syringe and mixed into soft food immediately before feeding to mice. 
Mineral oil treatment. Mice were administered mineral oil (food grade, CVS brand) or corn oil (Sigma, C8267) in soft food, mixed in as $0.1 \mathrm{~mL}$ oil per $1 \mathrm{~g}$ of food. The treatment was administered for 10 days, after which the animals were returned to regular solid pellets. Fecal pellets were collected for Lactobacillus level quantification, 5 days post treatment initiation and after 5 days of recovery. Escape behavior was measured as described above after 10 days of treatment. Food intake and weights were monitored and comparable throughout the experiment.

Drug administration. L-kynurenine (Sigma, K8625) was solubilized in water at a $4 \mathrm{mg} / \mathrm{mL}$ stock concentration. A 1:10 kynurenine working solution was prepared in sterile $0.9 \%$ saline solution and injected intraperitoneally at $2 \mathrm{mg} / \mathrm{kg}$ (approximately $0.15 \mathrm{~mL} /$ animal) for four weeks, daily. Injection site was alternated to avoid discomfort. An equivalent volume of saline solution was injected as control. For the IDO1 inhibitor experiment, 1-methyl tryptophan was purchased as D- (Sigma, 452483) and L- (Sigma, 447439) isomers and subsequently mixed stoichiometrically. Due to their poor solubility, and to avoid using organic solvents with potential effects on the microbiota, the drugs were thoroughly blended with pulverized dry food (at $1 \mathrm{mg} / \mathrm{g}$ each, equivalent to ${ }^{168}$ ) and subsequently mixed as soft food with water. The treatment was administered for three weeks, with the mice receiving fresh food every 1-2 days.

Intestinal transit time measurement. Mice were briefly anesthetized with isofluorane and a $3 \mathrm{~mm}$ diameter glass bead was inserted $2 \mathrm{~cm}$ inside the rectum with a vaseline lubricated rod. The mice were then placed in an empty cage without bedding and the time to bead expulsion was measured. 
Intestinal tissue analysis. The small intestine was dissected by excising under the stomach and before the cecum. Mesenteric fat and Peyer's patches were carefully removed using fine forceps. The intestine was opened longitudinally and the contents were removed in two PBS washes. Excess liquid was gently absorbed using kimwipes and the tissue was weighed. Intestinal cells were then isolated as previously described ${ }^{232}$. Briefly, the mucus layer and epithelial cells were shook off in two washes with HBSS / 5\% FBS/ 2 mM EDTA. The tissue was then digested with collagenase VIII (Sigma, C2139) for 12-15 minutes, filtered, and the cells washed twice with HBSS / 5\% FBS/ 2mM EDTA and finally resuspended in FACS buffer (0.01 M PBS, 1\% BSA, 2 mM EDTA, 0.1\% sodium azide). Cell counts and viability were determined using an acridine orange/propidium iodine assay on a Nexcelom cell counter. The cells were then stained for flow cytometry for 30 minutes, at $4{ }^{\circ} \mathrm{C}$ in the dark. After staining for extracellular antigens, the cells were fixed with either IC-Fix solution from eBioscience (for cytoplasmic antigens) or the BD fixation buffer (for intranuclear antigens) for 20 minutes, room temperature or overnight at $4^{\circ} \mathrm{C}$, respectively. The cells were washed with permeabilization buffer and further stained for intracellular antigens for 30 minutes, at $4^{\circ} \mathrm{C}$. Antibodies were generally obtained from either eBioscience or $\mathrm{BD}$, while the viability dyes and anti-Ly6G antibodies were purchased from Biolegend (clone information available upon request). After the final incubation, the cells were resuspended in $300 \mu \mathrm{L}$ PBS and subsequently analyzed on a Gallios flow cytometer (Beckman Coulter).

Metabolite analysis. Blood was collected by cardiac puncture and centrifuged at 10,000 $\mathrm{x}$ $\mathrm{g}$ for 3 minutes in gel tubes for serum preparation. Frozen $\left(-80^{\circ} \mathrm{C}\right)$ serum samples were shipped 
to the University of Michigan Metabolomics Core for untargeted metabolomics analysis. Metabolites isolated by positive and negative ion selection were analyzed by mass spectrometry. Mass spectrometry peak intensities were further analyzed using the MetaboAnalyst online software. Peak values were filtered using the interquantile range, normalized to the group sum, then log transformed and auto-scaled for principal component analysis and further statistical tests ${ }^{233}$. Identifiable significant metabolites were analyzed through pathway analysis, followed by further manual pathway enrichment.

ROS quantification. Fresh fecal samples were collected in sterile $2 \mathrm{~mL}$ tubes, weighed, and resuspended in $1 \mathrm{~mL}$ sterile PBS. After brief sedimentation of insoluble particles, $500 \mu \mathrm{L}$ of bacterial slurry were incubated at $37^{\circ} \mathrm{C}$ for 30 minutes. Following bacterial precipitation by centrifugation, $50 \mu \mathrm{L}$ of supernatant was reacted using the Amplex Red hydrogen peroxide/ peroxidase assay kit (Thermo Fisher, cat. A22188), according to manufacturer's protocol.

qPGR. Frozen tissues (brain and intestine) were homogenized by bead beating in RNA TRI Reagent (Life technologies) and RNA was extracted according to manufacturer's protocol. cDNA was synthethized with the High Capacity cDNA Reverse Transcription kit (Life Technologies). cDNA was amplified using the Sensifast Sybr NO-ROX kit (Bioline), according to manufacturer's instructions. Gapdh was measured as a normalizer for each sample. Results were analyzed by the relative quantity ( $\Delta \Delta \mathrm{Ct})$ method ${ }^{234}$. For total $16 \mathrm{~S}$ rRNA quantification, we followed the protocol described by Liu et al., ${ }^{235}$. L. reuteri DNA was used for the standard curve and specific primers for the $16 \mathrm{~S}$ rRNA V3-V4 region. For relative quantification of Lactobacillus, the $\Delta \Delta \mathrm{Ct}$ method was used to compare Lactobacillus-specific amplification to that 
of the 16S rRNA gene. Both reactions were performed using the Sensifast Sybr NoROX kit from Bioline (BIO-98005). Primer sequences are available in Table 1.

\begin{tabular}{|l|l|l|}
\hline Name & Forward seq & Reverse seq \\
\hline $\begin{array}{l}\text { 16S for library } \\
\text { with adapters } \\
\text { (per lllumina guide) }\end{array}$ & $\begin{array}{l}\text { TCGTCGGCAGCGTCAGATGTGTA } \\
\text { TAAGAGACAGCCTACGGGNGGCW }\end{array}$ & $\begin{array}{l}\text { GTCTCGTGGGCTCGGAGATGTGTA } \\
\text { TAAGAGACAGGACTACHVGGGTATC } \\
\text { TAATCC }\end{array}$ \\
\hline 16S & CCTACGGGDGGCWGCA & GGACTACHVGGGTMTCTAATC \\
\hline Lactobacillus & TGGATGCCTTGGCACTAGGA & AAATCTCCGGATCAAAGCTTACTTAT \\
\hline SFB & GACGCTGAGGCATGAGAGCAT & GACGGCACGGATTGTTATTCA \\
\hline L. reuteri & ACCTGATTGACGATGGATCACCAGT & CCACCTTCCTCCGGTTTGTCA \\
\hline ido1v1 & TGAAGATGTGGGCTTTGCTCT & TGTGGGCAGCTTTTCAACTTC \\
\hline
\end{tabular}

Table 1. List of primer sequences used in the study.

Statistical analysis. All statistical analyses were performed in Prism. Analyses involving two groups were performed using a two-tailed t-test. If the variances between groups were significantly different, a Welch correction was applied. For experiments involving stress and another variable (e.g. L.reuteri treatment or vendor), data were analyzed with a two-way ANOVA, followed by Bonferroni post-hoc tests. For the metabolomics experiments involving only 3 groups, a one-way ANOVA was utilized. Outliers were excluded if they fell more than two standard deviations from the mean. For all analyses, the threshold for significance was at $\mathrm{p}<0.05$. Repeats for each experiment, if performed, are specified in the figure legend corresponding to the respective panel. 


\section{Bibliography}

1 DSM-5. in Diagnostic and Statistical Manual of Mental Disorders DSM Library (American Psychiatric Association, 2013).

2 Montgomery, S. A. \& Asberg, M. A new depression scale designed to be sensitive to change., doi:10.1192/bjp.134.4.382 (1979).

3 Hamilton, M. A rating scale for depression. J Neurol Neurosurg Psychiatry 23, 5662 (1960).

4 Kessler, R. C. et al. The Epidemiology of Major Depressive Disorder: Results From the National Comorbidity Survey Replication (NCS-R). JAMA 289, 3095-3105, doi:10.1001/jama.289.23.3095 (2016).

5 Porsolt, R. D., Pichon, M. L. \& Jalfre, M. Depression: a new animal model sensitive to antidepressant treatments. 266, 730-732, doi:doi:10.1038/266730a0 (1977).

6 Cryan, J. F. \& Mombereau, C. In search of a depressed mouse: utility of models for studying depression-related behavior in genetically modified mice. Molecular Psychiatry 9, 326-357, doi:doi:10.1038/sj.mp.4001457 (2004).

7 Nollet, M., Le Guisquet, A. M. \& Belzung, C. Models of depression: unpredictable chronic mild stress in mice. Curr Protoc Pharmacol Chapter 5, Unit 5 65, doi:10.1002/0471141755.ph0565s61 (2013).

8 Nestler, E. J. \& Hyman, S. E. Animal models of neuropsychiatric disorders. Nature Neuroscience 13, 1161-1169, doi:doi:10.1038/nn.2647 (2010).

9 Kupfer, D. J., Frank, E. \& Phillips, M. L. Major depressive disorder: new clinical, neurobiological, and treatment perspectives. Lancet 379, 1045-1055, doi:10.1016/S0140-6736(11)60602-8 (2012).

10 Henn, F. A. \& Barbara, V. Stress models of depression: Forming genetically vulnerable strains. 29, 799-804, doi:10.1016/j.neubiorev.2005.03.019 (2005).

11 Lopez-Munoz, F. \& Alamo, C. Monoaminergic neurotransmission: the history of the discovery of antidepressants from 1950s until today. Curr Pharm Des 15, 15631586 (2009).

12 Cole, C. E. et al. A Controlled Study of Efficacy of Iproniazid in Treatment of Depression. A.M.A. Archives of General Psychiatry 1, 513-518, doi:10.1001/archpsyc.1959.03590050081010 (1959).

13 Kuhn, R. The treatment of depressive states with G 22355 (imipramine hydrochloride). Am J Psychiatry 115, 459-464, doi:10.1176/ajp.115.5.459 (1958). 
14 Krishnan, V. \& Nestler, E. J. The molecular neurobiology of depression. Nature 455, 894-902, doi:10.1038/nature07455 (2008).

15 Cerletti, A. \& Rothlin, E. Role of 5-Hydroxytryptamine in Mental Diseases and its Antagonism to Lysergic Acid Derivatives. 176, 785-786, doi:doi:10.1038/176785a0 (1955).

16 Woolley, D. W. \& Shaw, E. A BIOCHEMICAL AND PHARMACOLOGICAL SUGGESTION ABOUT CERTAIN MENTAL DISORDERS. Proc Natl Acad Sci U $S$ A 40, 228-231 (1954).

17 DeRubeis, R. J., Siegle, G. J. \& Hollon, S. D. Cognitive therapy versus medication for depression: treatment outcomes and neural mechanisms. Nature Reviews Neuroscience 9, 788-796, doi:doi:10.1038/nrn2345 (2008).

18 Cuijpers, P. et al. The effects of psychotherapies for major depression in adults on remission, recovery and improvement: a meta-analysis. J Affect Disord 159, 118126, doi:10.1016/j.jad.2014.02.026 (2014).

19 Ruhe, H. G., Mason, N. S. \& Schene, A. H. Mood is indirectly related to serotonin, norepinephrine and dopamine levels in humans: a meta-analysis of monoamine depletion studies. Mol Psychiatry 12, 331-359, doi:10.1038/sj.mp.4001949 (2007).

20 Lee, H., Ohno, M., Ohta, S. \& Mikami, T. Regular moderate or intense exercise prevents depression-like behavior without change of hippocampal tryptophan content in chronically tryptophan-deficient and stressed mice. PLoS One 8, e66996, doi:10.1371/journal.pone.0066996 (2013).

21 Franklin, M., Bermudez, I., Murck, H., Singewald, N. \& Gaburro, S. Sub-chronic dietary tryptophan depletion--an animal model of depression with improved face and good construct validity. $J$ Psychiatr Res 46, 239-247, doi:10.1016/j.jpsychires.2011.10.003 (2012).

22 Cahir, M., Ardis, T., Reynolds, G. P. \& Cooper, S. J. Acute and chronic tryptophan depletion differentially regulate central $5-\mathrm{HT} 1 \mathrm{~A}$ and $5-\mathrm{HT} 2 \mathrm{~A}$ receptor binding in the rat. Psychopharmacology (Berl) 190, 497-506, doi:10.1007/s00213-006-06355 (2007).

23 Krishnan, V. et al. Molecular adaptations underlying susceptibility and resistance to social defeat in brain reward regions. Cell 131, 391-404, doi:10.1016/j.cell.2007.09.018 (2007).

24 Chaudhury, D. et al. Rapid regulation of depression-related behaviours by control of midbrain dopamine neurons. Nature 493, 532-536, doi:10.1038/nature11713 (2013).

25 Friedman, A. K. et al. Enhancing depression mechanisms in midbrain dopamine neurons achieves homeostatic resilience. Science 344, 313-319, doi:10.1126/science.1249240 (2014). 
26 Duman, R. S. \& Aghajanian, G. K. Synaptic Dysfunction in Depression: Potential Therapeutic Targets. doi:10.1126/science.1222939 (2012).

27 Cai, X. et al. Local potentiation of excitatory synapses by serotonin and its alteration in rodent models of depression. Nat Neurosci 16, 464-472, doi:10.1038/nn.3355 (2013).

28 Monteggia, L. M., Malenka, R. C. \& Deisseroth, K. Depression: The best way forward. Nature 515, 200-201, doi:doi:10.1038/515200a (2014).

29 Domino, E. F. et al. Pharmacologic effects of Cl-581, a new dissociative anesthetic, in man. Clinical Pharmacology \& Therapeutics 6, 279-291, doi:10.1002/cpt196563279 (2016).

30 Berman, R. M. et al. Antidepressant effects of ketamine in depressed patients. 47, 351-354, doi:10.1016/S0006-3223(99)00230-9 (2000).

31 Newport, D. J. et al. Ketamine and Other NMDA Antagonists: Early Clinical Trials and Possible Mechanisms in Depression. Am J Psychiatry 172, 950-966, doi:10.1176/appi.ajp.2015.15040465 (2015).

32 Jafarinia, M. et al. Efficacy and safety of oral ketamine versus diclofenac to alleviate mild to moderate depression in chronic pain patients: A double-blind, randomized, controlled trial. J Affect Disord 204, 1-8, doi:10.1016/j.jad.2016.05.076 (2016).

33 Autry, A. E. et al. NMDA receptor blockade at rest triggers rapid behavioural antidepressant responses. Nature 475, 91-95, doi:10.1038/nature10130 (2011).

34 Miller, O. H. et al. GluN2B-containing NMDA receptors regulate depression-like behavior and are critical for the rapid antidepressant actions of ketamine. Elife 3, e03581, doi:10.7554/eLife.03581 (2014).

35 Zanos, P. et al. NMDAR inhibition-independent antidepressant actions of ketamine metabolites. Nature 533, 481-486, doi:10.1038/nature17998 (2016).

36 Li, Y. X., Zhang, Y., Lester, H. A., Schuman, E. M. \& Davidson, N. Enhancement of neurotransmitter release induced by brain-derived neurotrophic factor in cultured hippocampal neurons. J Neurosci 18, 10231-10240 (1998).

37 Tyler, W. J. \& Pozzo-Miller, L. D. BDNF enhances quantal neurotransmitter release and increases the number of docked vesicles at the active zones of hippocampal excitatory synapses. $J$ Neurosci 21, 4249-4258 (2001).

38 Jovanovic, J. N., Czernik, A. J., Fienberg, A. A., Greengard, P. \& Sihra, T. S. Synapsins as mediators of BDNF-enhanced neurotransmitter release. Nat Neurosci 3, 323-329, doi:10.1038/73888 (2000).

39 Figurov, A., Pozzo-Miller, L. D., Olafsson, P., Wang, T. \& Lu, B. Regulation of synaptic responses to high-frequency stimulation and LTP by neurotrophins in the hippocampus. Nature 381, 706-709, doi:10.1038/381706a0 (1996). 
40 Monteggia, L. M. et al. Brain-derived neurotrophic factor conditional knockouts show gender differences in depression-related behaviors. Biol Psychiatry 61, 187197, doi:10.1016/j.biopsych.2006.03.021 (2007).

41 Autry, A. E., Adachi, M., Cheng, P. \& Monteggia, L. M. Gender-specific impact of brain-derived neurotrophic factor signaling on stress-induced depression-like behavior. Biol Psychiatry 66, 84-90, doi:10.1016/j.biopsych.2009.02.007 (2009).

42 Caffino, L. et al. The modulation of BDNF expression and signalling dissects the antidepressant from the reinforcing properties of ketamine: Effects of single infusion vs. chronic self-administration in rats. Pharmacol Res 104, 22-30, doi:10.1016/j.phrs.2015.12.014 (2016).

43 Holsboer, F. The corticosteroid receptor hypothesis of depression. Neuropsychopharmacology 23, 477-501, doi:10.1016/s0893-133x(00)00159-7 (2000).

44 Pariante, C. M. \& Lightman, S. L. The HPA axis in major depression: classical theories and new developments. Trends in Neurosciences 31, 464-468, doi:10.1016/j.tins.2008.06.006 (2008).

45 Lehmann, M. L., Brachman, R. A., Martinowich, K., Schloesser, R. J. \& Herkenham, M. Glucocorticoids orchestrate divergent effects on mood through adult neurogenesis. J Neurosci 33, 2961-2972, doi:10.1523/jneurosci.387812.2013 (2013).

46 de Quervain, D. J., Roozendaal, B. \& McGaugh, J. L. Stress and glucocorticoids impair retrieval of long-term spatial memory. Nature 394, 787-790, doi:10.1038/29542 (1998).

47 Gold, P. W. et al. Responses to corticotropin-releasing hormone in the hypercortisolism of depression and Cushing's disease. Pathophysiologic and diagnostic implications. $N$ Engl $J$ Med 314, 1329-1335, doi:10.1056/nejm198605223142101 (1986).

48 Ising, M. et al. Combined dexamethasone/corticotropin releasing hormone test predicts treatment response in major depression - a potential biomarker? Biol Psychiatry 62, 47-54, doi:10.1016/j.biopsych.2006.07.039 (2007).

49 Mokhtari, M., Arfken, C. \& Boutros, N. The DEX/CRH test for major depression: a potentially useful diagnostic test. Psychiatry Res 208, 131-139, doi:10.1016/j.psychres.2012.09.032 (2013).

50 Schüle, C. et al. in PLoS ONE Vol. 4 (2009).

51 Blasey, C. M., Block, T. S., Belanoff, J. K. \& Roe, R. L. Efficacy and safety of mifepristone for the treatment of psychotic depression. J Clin Psychopharmacol 31, 436-440, doi:10.1097/JCP.0b013e3182239191 (2011).

52 Gallagher, P. \& Young, A. H. in Neuropsychiatr Dis Treat Vol. 2 33-42 (2006). 
53 Chen, D. Y., Bambah-Mukku, D., Pollonini, G. \& Alberini, C. M. Glucocorticoid receptors recruit the CaMKIIalpha-BDNF-CREB pathways to mediate memory consolidation. Nat Neurosci 15, 1707-1714, doi:10.1038/nn.3266 (2012).

54 Sarabdjitsingh, R. A. et al. Ultradian corticosterone pulses balance glutamatergic transmission and synaptic plasticity. Proc Natl Acad Sci U S A 111, 14265-14270, doi:10.1073/pnas.1411216111 (2014).

55 Liston, C. \& Gan, W. B. Glucocorticoids are critical regulators of dendritic spine development and plasticity in vivo. Proc Natl Acad Sci U S A 108, 16074-16079, doi:10.1073/pnas.1110444108 (2011).

56 Lemos, J. C. et al. Severe stress switches CRF action in the nucleus accumbens from appetitive to aversive. Nature 490, 402-406, doi:10.1038/nature11436

nature11436 [pii] (2012).

57 Radley, J. J. et al. Repeated stress induces dendritic spine loss in the rat medial prefrontal cortex. Cereb Cortex 16, 313-320, doi:10.1093/cercor/bhi104 (2006).

58 Arango-Lievano, M. et al. Neurotrophic-priming of glucocorticoid receptor signaling is essential for neuronal plasticity to stress and antidepressant treatment. Proc Natl Acad Sci U S A 112, 15737-15742, doi:10.1073/pnas.1509045112 (2015).

59 Rozeboom, A. M., Akil, H. \& Seasholtz, A. F. Mineralocorticoid receptor overexpression in forebrain decreases anxiety-like behavior and alters the stress response in mice. Proc Natl Acad Sci U S A 104, 4688-4693, doi:10.1073/pnas.0606067104 (2007).

60 Ridder, S. et al. Mice with genetically altered glucocorticoid receptor expression show altered sensitivity for stress-induced depressive reactions. J Neurosci 25, 6243-6250, doi:10.1523/jneurosci.0736-05.2005 (2005).

61 Wei, Q. et al. Glucocorticoid receptor overexpression in forebrain: a mouse model of increased emotional lability. Proc Natl Acad Sci U S A 101, 11851-11856, doi:10.1073/pnas.0402208101 (2004).

62 Boyle, M. P. et al. Acquired deficit of forebrain glucocorticoid receptor produces depression-like changes in adrenal axis regulation and behavior. Proc Natl Acad Sci U S A 102, 473-478, doi:10.1073/pnas.0406458102 (2005).

63 Snyder, J. S., Soumier, A., Brewer, M., Pickel, J. \& Cameron, H. A. Adult hippocampal neurogenesis buffers stress responses and depressive behaviour. Nature 476, 458-461, doi:10.1038/nature10287 (2011).

64 Steinberg, E. E., Christoffel, D. J., Deisseroth, K. \& Malenka, R. C. Illuminating circuitry relevant to psychiatric disorders with optogenetics. Curr Opin Neurobiol 30, 9-16, doi:10.1016/j.conb.2014.08.004 (2015).

65 Russo, S. J. \& Nestler, E. J. The brain reward circuitry in mood disorders. Nature Reviews Neuroscience 14, 609-625, doi:doi:10.1038/nrn3381 (2013). 
66 Hong, S. \& Hikosaka, O. The globus pallidus sends reward-related signals to the lateral habenula. Neuron 60, 720-729, doi:10.1016/j.neuron.2008.09.035 (2008).

67 Matsumoto, M. \& Hikosaka, O. Lateral habenula as a source of negative reward signals in dopamine neurons. Nature 447, 1111-1115, doi:10.1038/nature05860 (2007).

68 Matsumoto, M. \& Hikosaka, O. Representation of negative motivational value in the primate lateral habenula. Nat Neurosci 12, 77-84, doi:10.1038/nn.2233 (2009).

69 Boulos, L.-J., Darcq, E., Kieffer, B. L. \& brigitte.kieffer@douglas.mcgill.ca. Translating the Habenula-From Rodents to Humans. Biological Psychiatry 0, doi:10.1016/j.biopsych.2016.06.003 (2016).

$70 \mathrm{Li}, \mathrm{B}$. et al. Synaptic potentiation onto habenula neurons in the learned helplessness model of depression. Nature 470, 535-539, doi:doi:10.1038/nature09742 (2011).

71 Caldecott-Hazard, S., Mazziotta, J. \& Phelps, M. Cerebral correlates of depressed behavior in rats, visualized using 14C-2-deoxyglucose autoradiography. $J$ Neurosci 8, 1951-1961 (1988).

72 Shabel, S. J., Proulx, C. D., Piriz, J. \& Malinow, R. Mood regulation. GABA/glutamate co-release controls habenula output and is modified by antidepressant treatment. Science 345, 1494-1498, doi:10.1126/science.1250469 (2014).

73 Krishnan, V. et al. AKT signaling within the ventral tegmental area regulates cellular and behavioral responses to stressful stimuli. Biol Psychiatry 64, 691-700, doi:10.1016/j.biopsych.2008.06.003 (2008).

74 Tye, K. M. et al. Dopamine neurons modulate neural encoding and expression of depression-related behaviour. Nature 493, 537-541, doi:10.1038/nature11740 (2013).

75 Sullivan, P. F., Neale, M. C. \& Kendler, K. S. Genetic Epidemiology of Major Depression: Review and Meta-Analysis. http://dx.doi.org/10.1176/appi.ajp.157.10.1552, doi:1552.pdf (2000).

76 Piccinelli, M. \& Wilkinson, G. Gender differences in depression. doi:10.1192/bjp.177.6.486 (2000).

77 Hind, D. et al. The assessment of depression in people with multiple sclerosis: a systematic review of psychometric validation studies. BMC Psychiatry 16, 1, doi:10.1186/s12888-016-0931-5 (2016).

78 Siegert, R. J. \& Abernethy, D. A. Depression in multiple sclerosis: a review. doi:10.1136/jnnp.2004.054635 (2005).

79 Miller, A. H. \& Raison, C. L. The role of inflammation in depression: from evolutionary imperative to modern treatment target. Nature Reviews Immunology 16, 22-34, doi:doi:10.1038/nri.2015.5 (2016). 
80 Wohleb, E. S., Franklin, T., Iwata, M. \& Duman, R. S. Integrating neuroimmune systems in the neurobiology of depression. Nature Reviews Neuroscience 17, 497511, doi:doi:10.1038/nrn.2016.69 (2016).

81 Dantzer, R. \& Kelley, K. W. Twenty years of research on cytokine-induced sickness behavior. Brain Behav Immun 21, 153-160, doi:10.1016/j.bbi.2006.09.006 (2007).

82 Marin, I. \& Kipnis, J. Learning and memory ... and the immune system. Learn Mem 20, 601-606, doi:10.1101//m.028357.112

20/10/601 [pii] (2013).

83 Marin, I. A. \& Kipnis, J. Central Nervous System: (Immunological) Ivory Tower or Notl[quest]. Neuropsychopharmacology : official publication of the American College of Neuropsychopharmacology, doi:doi:10.1038/npp.2016.122 (2016).

84 Agudelo, L. Z. et al. Skeletal muscle PGC-1alpha1 modulates kynurenine metabolism and mediates resilience to stress-induced depression. Cell 159, 3345, doi:10.1016/j.cell.2014.07.051 (2014).

85 Pan, L. A. et al. Neurometabolic Disorders: Potentially Treatable Abnormalities in Patients With Treatment-Refractory Depression and Suicidal Behavior. Am J Psychiatry, appiajp201615111500, doi:10.1176/appi.ajp.2016.15111500 (2016).

86 Petridou, E. T. et al. Folate and B12 serum levels in association with depression in the aged: a systematic review and meta-analysis. Aging Ment Health 20, 965973, doi:10.1080/13607863.2015.1049115 (2016).

87 Labouebe, G. et al. Insulin induces long-term depression of ventral tegmental area dopamine neurons via endocannabinoids. Nat Neurosci 16, 300-308, doi:10.1038/nn.3321 (2013).

88 Harris, A. H., Cronkite, R. \& Moos, R. Physical activity, exercise coping, and depression in a 10-year cohort study of depressed patients. J Affect Disord 93, 7985, doi:10.1016/j.jad.2006.02.013 (2006).

89 Sahay, A. \& Hen, R. Adult hippocampal neurogenesis in depression. Nat Neurosci 10, 1110-1115, doi:10.1038/nn1969 (2007).

90 Capuron, L., Lasselin, J. \& Castanon, N. Role of Adiposity-Driven Inflammation in Depressive Morbidity. Neuropsychopharmacology, doi:doi:10.1038/npp.2016.123 (2016).

91 Murphy, P., Likhodii, S., Nylen, K. \& Burnham, W. M. The antidepressant properties of the ketogenic diet. Biol Psychiatry 56, 981-983, doi:10.1016/j.biopsych.2004.09.019 (2004).

92 Koranda, J. L., Ruskin, D. N., Masino, S. A. \& Blaise, J. H. A ketogenic diet reduces long-term potentiation in the dentate gyrus of freely behaving rats. $J$ Neurophysiol 106, 662-666, doi:10.1152/jn.00001.2011 (2011). 
93 Foster, J. A. \& McVey Neufeld, K. A. Gut-brain axis: how the microbiome influences anxiety and depression. Trends Neurosci 36, 305-312, doi:10.1016/j.tins.2013.01.005

S0166-2236(13)00008-8 [pii] (2013).

94 Gilbert, J. A. et al. Microbiome-wide association studies link dynamic microbial consortia to disease. Nature 535, 94-103, doi:10.1038/nature18850 (2016).

95 Sonnenburg, J. L. \& Backhed, F. Diet-microbiota interactions as moderators of human metabolism. Nature 535, 56-64, doi:10.1038/nature18846 (2016).

96 Hooper, L., Littman, D. \& Macpherson, A. Interactions between the microbiota and the immune system. Science (New York, N.Y.) 336, 1268-1273, doi:10.1126/science.1223490 (2012).

97 Bonaz, B., Picq, C., Sinniger, V., Mayol, J. F. \& Clarencon, D. Vagus nerve stimulation: from epilepsy to the cholinergic anti-inflammatory pathway. Neurogastroenterol Motil 25, 208-221, doi:10.1111/nmo.12076 (2013).

98 Prechtl, J. C. \& Powley, T. L. The fiber composition of the abdominal vagus of the rat. Anat Embryol (Berl) 181, 101-115 (1990).

99 Berthoud, H. R. \& Neuhuber, W. L. Functional and chemical anatomy of the afferent vagal system. Auton Neurosci 85, 1-17, doi:S1566-0702(00)00215-0 [pii]

10.1016/S1566-0702(00)00215-0 (2000).

100 Straub, R. H., Wiest, R., Strauch, U. G., Harle, P. \& Scholmerich, J. The role of the sympathetic nervous system in intestinal inflammation. Gut 55, 1640-1649, doi:55/11/1640 [pii]

10.1136/gut.2006.091322 (2006).

101 Dethlefsen, L., Eckburg, P. B., Bik, E. M. \& Relman, D. A. Assembly of the human intestinal microbiota. Trends Ecol Evol 21, 517-523, doi:S0169-5347(06)00201-1 [pii]

10.1016/j.tree.2006.06.013 (2006).

102 Nicholson, J. K. et al. Host-gut microbiota metabolic interactions. Science 336, 1262-1267, doi:10.1126/science.1223813

science.1223813 [pii] (2012).

103 Torrey, J. C. The Regulation of the Intestinal Flora of Dogs through Diet. J Med Res 39, 415-447 (1919).

104 Wostmann, B. S., Larkin, C., Moriarty, A. \& Bruckner-Kardoss, E. Dietary intake, energy metabolism, and excretory losses of adult male germfree Wistar rats. Lab Anim Sci 33, 46-50 (1983). 
105 Tamburini, S., Shen, N., Wu, H. C. \& Clemente, J. C. The microbiome in early life: implications for health outcomes. Nat Med 22, 713-722, doi:10.1038/nm.4142 (2016).

106 Ravel, J. et al. Vaginal microbiome of reproductive-age women. Proc Natl Acad Sci U S A 108 Suppl 1, 4680-4687, doi:10.1073/pnas.1002611107 (2011).

107 Campbell, J. H. et al. Host genetic and environmental effects on mouse intestinal microbiota. The ISME Journal 6, 2033-2044, doi:doi:10.1038/ismej.2012.54 (2012).

108 Xiao, L. et al. A catalog of the mouse gut metagenome. Nature Biotechnology 33, 1103-1108, doi:doi:10.1038/nbt.3353 (2015).

109 Arrieta, M. C., Walter, J. \& Finlay, B. B. Human Microbiota-Associated Mice: A Model with Challenges. Cell Host Microbe 19, 575-578, doi:10.1016/j.chom.2016.04.014 (2016).

110 Smith, M. I. et al. Gut Microbiomes of Malawian Twin Pairs Discordant for Kwashiorkor. doi:10.1126/science.1229000 (2013).

111 Zheng, P. et al. Gut microbiome remodeling induces depressive-like behaviors through a pathway mediated by the host's metabolism. Mol Psychiatry 21, 786796, doi:10.1038/mp.2016.44 (2016).

112 Biotech, E. N. Laying better plans for mice. Nat Biotech 31, 263-263, doi:10.1038/nbt.2560 (2013).

113 Bäumler, A. J. \& Sperandio, V. Interactions between the microbiota and pathogenic bacteria in the gut. Nature 535, 85-93, doi:doi:10.1038/nature18849 (2016).

114 Thaiss, C. A., Zmora, N., Levy, M. \& Elinav, E. The microbiome and innate immunity. Nature 535, 65-74, doi:10.1038/nature18847 (2016).

115 Honda, K. \& Littman, D. R. The microbiota in adaptive immune homeostasis and disease. Nature 535, 75-84, doi:10.1038/nature18848 (2016).

116 Erny, D. et al. Host microbiota constantly control maturation and function of microglia in the CNS. Nature Neuroscience 18, 965-977, doi:doi:10.1038/nn.4030 (2015).

117 Macpherson, A. J. \& Uhr, T. Induction of protective IgA by intestinal dendritic cells carrying commensal bacteria. Science 303, 1662-1665, doi:10.1126/science.1091334

303/5664/1662 [pii] (2004).

118 Sonnenberg, G. F. et al. Innate lymphoid cells promote anatomical containment of lymphoid-resident commensal bacteria. Science 336, 1321-1325, doi:10.1126/science.1222551

science.1222551 [pii] (2012). 
119 Elinav, E. et al. NLRP6 inflammasome regulates colonic microbial ecology and risk for colitis. Cell 145, 745-757, doi:10.1016/j.cell.2011.04.022

S0092-8674(11)00480-6 [pii] (2011).

120 Vijay-Kumar, M. et al. Metabolic syndrome and altered gut microbiota in mice lacking Toll-like receptor 5. Science 328, 228-231, doi:10.1126/science.1179721

science.1179721 [pii] (2010).

121 Garrett, W. S. et al. Communicable ulcerative colitis induced by T-bet deficiency in the innate immune system. Cell 131, 33-45, doi:S0092-8674(07)01080-X [pii]

10.1016/j.cell.2007.08.017 (2007).

122 Ohwaki, M., Yasutake, N., Yasui, H. \& Ogura, R. A comparative study on the humoral immune responses in germ-free and conventional mice. Immunology $\mathbf{3 2}$, 43-48 (1977).

123 Mazmanian, S. K., Liu, C. H., Tzianabos, A. O. \& Kasper, D. L. An immunomodulatory molecule of symbiotic bacteria directs maturation of the host immune system. Cell 122, 107-118, doi:S0092-8674(05)00451-4 [pii]

10.1016/j.cell.2005.05.007 (2005).

124 Chung, $\mathrm{H}$. et al. Gut immune maturation depends on colonization with a hostspecific microbiota. Cell 149, 1578-1593, doi:10.1016/j.cell.2012.04.037

S0092-8674(12)00629-0 [pii] (2012).

125 Olszak, T. et al. Microbial exposure during early life has persistent effects on natural killer T cell function. Science 336, 489-493, doi:10.1126/science.1219328

science.1219328 [pii] (2012).

126 Ivanov, II et al. Induction of intestinal Th17 cells by segmented filamentous bacteria. Cell 139, 485-498, doi:10.1016/j.cell.2009.09.033

S0092-8674(09)01248-3 [pii] (2009).

127 Surana, N. K. \& Kasper, D. L. The yin yang of bacterial polysaccharides: lessons learned from B. fragilis PSA. Immunol Rev 245, 13-26, doi:10.1111/j.1600065X.2011.01075.X (2012).

128 Atarashi, K. et al. Induction of colonic regulatory T cells by indigenous Clostridium species. Science 331, 337-341, doi:10.1126/science.1198469

science.1198469 [pii] (2011).

129 Atarashi, K. et al. Treg induction by a rationally selected mixture of Clostridia strains from the human microbiota. Nature 500, 232-236, doi:10.1038/nature12331

nature12331 [pii] (2013). 
130 Cryan, J. F. \& Dinan, T. G. Mind-altering microorganisms: the impact of the gut microbiota on brain and behaviour. Nat Rev Neurosci 13, 701-712, doi:10.1038/nrn3346

nrn3346 [pii] (2012).

131 Dinan, T. G. \& Cryan, J. F. Regulation of the stress response by the gut microbiota: implications for psychoneuroendocrinology. Psychoneuroendocrinology 37, 13691378, doi:10.1016/j.psyneuen.2012.03.007

S0306-4530(12)00093-5 [pii] (2012).

132 Rogers, G. B. et al. From gut dysbiosis to altered brain function and mental illness: mechanisms and pathways. Mol Psychiatry 21, 738-748, doi:10.1038/mp.2016.50 (2016).

133 Kang, D. W. et al. Reduced incidence of Prevotella and other fermenters in intestinal microflora of autistic children. PLoS One 8, e68322, doi:10.1371/journal.pone.0068322

PONE-D-13-03000 [pii] (2013).

134 Severance, E. G. et al. Gastrointestinal inflammation and associated immune activation in schizophrenia. Schizophr Res 138, 48-53, doi:10.1016/j.schres.2012.02.025

S0920-9964(12)00147-8 [pii] (2012).

135 Cremonini, F. et al. Sleep disturbances are linked to both upper and lower gastrointestinal symptoms in the general population. Neurogastroenterol Motil 21, 128-135, doi:10.1111/j.1365-2982.2008.01181.x

NMO1181 [pii] (2009).

136 Naseribafrouei, A. et al. Correlation between the human fecal microbiota and depression. Neurogastroenterol Motil 26, 1155-1162, doi:10.1111/nmo.12378 (2014).

137 Jiang, $\mathrm{H}$. et al. Altered fecal microbiota composition in patients with major depressive disorder. Brain Behav Immun 48, 186-194, doi:10.1016/j.bbi.2015.03.016 (2015).

138 Adams, J. B., Johansen, L. J., Powell, L. D., Quig, D. \& Rubin, R. A. Gastrointestinal flora and gastrointestinal status in children with autism-comparisons to typical children and correlation with autism severity. BMC Gastroenterol 11, 22, doi:10.1186/1471-230x-11-22 (2011).

139 Williams, B. L. et al. Impaired carbohydrate digestion and transport and mucosal dysbiosis in the intestines of children with autism and gastrointestinal disturbances. PLoS One 6, e24585, doi:10.1371/journal.pone.0024585 (2011). 
140 Diaz Heijtz, R. et al. Normal gut microbiota modulates brain development and behavior. Proceedings of the National Academy of Sciences of the United States of America 108, 3047-3052, doi:10.1073/pnas.1010529108 (2011).

141 Desbonnet, L. et al. Gut microbiota depletion from early adolescence in mice: Implications for brain and behaviour. Brain Behav Immun 48, 165-173, doi:10.1016/j.bbi.2015.04.004 (2015).

142 Bercik, P. et al. The intestinal microbiota affect central levels of brain-derived neurotropic factor and behavior in mice. Gastroenterology 141, 599-609, 609 e591-593, doi:10.1053/j.gastro.2011.04.052

S0016-5085(11)00607-X [pii] (2011).

143 Sudo, N. et al. Postnatal microbial colonization programs the hypothalamicpituitary-adrenal system for stress response in mice. The Journal of physiology 558, 263-275, doi:10.1113/jphysiol.2004.063388 (2004).

144 Clarke, G. et al. The microbiome-gut-brain axis during early life regulates the hippocampal serotonergic system in a sex-dependent manner. Mol Psychiatry 18, 666-673, doi:10.1038/mp.2012.77

mp201277 [pii] (2013).

145 Buffington, S. A. et al. Microbial Reconstitution Reverses Maternal Diet-Induced Social and Synaptic Deficits in Offspring. Cell 165, 1762-1775, doi:10.1016/j.cell.2016.06.001 (2016).

146 Braniste, V. et al. The gut microbiota influences blood-brain barrier permeability in mice. Sci Transl Med 6, 263ra158, doi:10.1126/scitranslmed.3009759 (2014).

147 Gareau, M. G., Jury, J., MacQueen, G., Sherman, P. M. \& Perdue, M. H. Probiotic treatment of rat pups normalises corticosterone release and ameliorates colonic dysfunction induced by maternal separation. Gut 56, 1522-1528, doi:gut.2006.117176 [pii]

10.1136/gut.2006.117176 (2007).

148 Barouei, J., Moussavi, M. \& Hodgson, D. M. Effect of maternal probiotic intervention on HPA axis, immunity and gut microbiota in a rat model of irritable bowel syndrome. PLoS One 7, e46051, doi:10.1371/journal.pone.0046051

PONE-D-12-16517 [pii] (2012).

149 Galley, J. D. et al. Exposure to a social stressor disrupts the community structure of the colonic mucosa-associated microbiota. BMC Microbiol 14, 189, doi:10.1186/1471-2180-14-189 (2014).

150 De Palma, G. et al. Microbiota and host determinants of behavioural phenotype in maternally separated mice. Nature communications 6, 7735, doi:10.1038/ncomms8735 (2015). 
151 Jasarevic, E., Howerton, C. L., Howard, C. D. \& Bale, T. L. Alterations in the Vaginal Microbiome by Maternal Stress Are Associated With Metabolic Reprogramming of the Offspring Gut and Brain. Endocrinology 156, 3265-3276, doi:10.1210/en.2015-1177 (2015).

152 Gacias, M. et al. Microbiota-driven transcriptional changes in prefrontal cortex override genetic differences in social behavior. Elife 5, doi:10.7554/eLife.13442 (2016).

153 Hsiao, E. Y. et al. Microbiota modulate behavioral and physiological abnormalities associated with neurodevelopmental disorders. Cell 155, 1451-1463, doi:10.1016/j.cell.2013.11.024 (2013).

154 Willner, P. Validity, reliability and utility of the chronic mild stress model of depression: a 10-year review and evaluation. Psychopharmacology (Berl) 134, 319-329 (1997).

155 Mineur, Y. S., Belzung, C. \& Crusio, W. E. Effects of unpredictable chronic mild stress on anxiety and depression-like behavior in mice. Behav Brain Res 175, 4350, doi:10.1016/j.bbr.2006.07.029 (2006).

$156 \mathrm{Li}, \mathrm{X}$., Morrow, D. \& Witkin, J. M. Decreases in nestlet shredding of mice by serotonin uptake inhibitors: comparison with marble burying. Life Sci 78, 19331939, doi:10.1016/j.Ifs.2005.08.002 (2006).

157 Hoy, Y. E. et al. Variation in Taxonomic Composition of the Fecal Microbiota in an Inbred Mouse Strain across Individuals and Time. PLoS One 10, e0142825, doi:10.1371/journal.pone.0142825 (2015).

158 Ericsson, A. C. et al. Effects of vendor and genetic background on the composition of the fecal microbiota of inbred mice. PLoS One 10, e0116704, doi:10.1371/journal.pone.0116704 (2015).

159 Liu, Y. et al. Actions of hydrogen sulfide and ATP-sensitive potassium channels on colonic hypermotility in a rat model of chronic stress. PLoS One 8, e55853, doi:10.1371/journal.pone.0055853 (2013).

160 Bunnett, N. W. The stressed gut: contributions of intestinal stress peptides to inflammation and motility. Proc Natl Acad Sci US A 102, 7409-7410, doi:10.1073/pnas.0503092102 (2005).

161 Altermann, E. et al. Complete genome sequence of the probiotic lactic acid bacterium Lactobacillus acidophilus NCFM. Proc Natl Acad Sci U S A 102, 39063912, doi:10.1073/pnas.0409188102 (2005).

162 Kant, R., Blom, J., Palva, A., Siezen, R. J. \& de Vos, W. M. Comparative genomics of Lactobacillus. Microb Biotechnol 4, 323-332, doi:10.1111/j.17517915.2010.00215.x (2011). 
163 Morita, $\mathrm{H}$. et al. Complete genome sequence of the probiotic Lactobacillus rhamnosus ATCC 53103. J Bacteriol 191, 7630-7631, doi:10.1128/jb.01287-09 (2009).

164 Pridmore, R. D. et al. The genome sequence of the probiotic intestinal bacterium Lactobacillus johnsonii NCC 533. Proc Natl Acad Sci U S A 101, 2512-2517 (2004).

165 Oh, P. L. et al. Diversification of the gut symbiont Lactobacillus reuteri as a result of host-driven evolution. ISME J 4, 377-387, doi:10.1038/ismej.2009.123 (2010).

166 Valladares, R. et al. Lactobacillus johnsonii inhibits indoleamine 2,3-dioxygenase and alters tryptophan metabolite levels in BioBreeding rats. FASEB $J$ 27, 17111720, doi:10.1096/fj.12-223339 (2013).

167 Schwarcz, R., Bruno, J. P., Muchowski, P. J. \& Wu, H. Q. Kynurenines in the mammalian brain: when physiology meets pathology. Nat Rev Neurosci 13, 465477, doi:10.1038/nrn3257 (2012).

168 Hou, D.-Y. et al. Inhibition of Indoleamine 2,3-Dioxygenase in Dendritic Cells by Stereoisomers of 1-Methyl-Tryptophan Correlates with Antitumor Responses. doi:10.1158/0008-5472.CAN-06-2925 (2007).

169 Pigott, E. et al. 1-Methyl-Tryptophan Synergizes with Methotrexate to Alleviate Arthritis in a Mouse Model of Arthritis. Autoimmunity 47, 409-418, doi:10.3109/08916934.2014.914507 (2014).

170 Brand, M. W. et al. The Altered Schaedler Flora: Continued Applications of a Defined Murine Microbial Community. doi:10.1093/ilar/ilv012 (2015).

171 Dewhirst, F. E. et al. Phylogeny of the Defined Murine Microbiota: Altered Schaedler Flora. (1999).

172 Myint, A. M. et al. Kynurenine pathway in major depression: evidence of impaired neuroprotection. J Affect Disord 98, 143-151, doi:10.1016/j.jad.2006.07.013 (2007).

173 O'Connor, J. C. et al. Lipopolysaccharide-induced depressive-like behavior is mediated by indoleamine 2,3-dioxygenase activation in mice. Mol Psychiatry 14, 511-522, doi:10.1038/sj.mp.4002148 (2009).

174 Fuertig, R. et al. Mouse chronic social stress increases blood and brain kynurenine pathway activity and fear behaviour: Both effects are reversed by inhibition of indoleamine 2,3-dioxygenase. Brain Behav Immun 54, 59-72, doi:10.1016/j.bbi.2015.12.020 (2016).

175 Jasarevic, E., Rodgers, A. B. \& Bale, T. L. A novel role for maternal stress and microbial transmission in early life programming and neurodevelopment. Neurobiology of stress 1, 81-88, doi:10.1016/j.ynstr.2014.10.005 (2015).

176 O'Callaghan, J. \& O'Toole, P. W. Lactobacillus: host-microbe relationships. Curr Top Microbiol Immunol 358, 119-154, doi:10.1007/82_2011_187 (2013). 
177 Gil, N. F., Martinez, R. C., Gomes, B. C., Nomizo, A. \& De Martinis, E. C. Vaginal lactobacilli as potential probiotics against Candida SPP. Braz J Microbiol 41, 6-14, doi:10.1590/S1517-83822010000100002 (2010).

178 Hertzberger, R. et al. $\mathrm{H}(2) \mathrm{O}(2)$ production in species of the Lactobacillus acidophilus group: a central role for a novel NADH-dependent flavin reductase. Appl Environ Microbiol 80, 2229-2239, doi:10.1128/AEM.04272-13 (2014).

179 Badet, C. \& Thebaud, N. B. Ecology of lactobacilli in the oral cavity: a review of literature. Open Microbiol J 2, 38-48, doi:10.2174/1874285800802010038 (2008).

180 Cani, P. D. \& Van Hul, M. Novel opportunities for next-generation probiotics targeting metabolic syndrome. Curr Opin Biotechnol 32, 21-27, doi:10.1016/j.copbio.2014.10.006 (2015).

181 Walter, J., Britton, R. A. \& Roos, S. Host-microbial symbiosis in the vertebrate gastrointestinal tract and the Lactobacillus reuteri paradigm. Proc Natl Acad Sci U S A 108 Suppl 1, 4645-4652, doi:10.1073/pnas.1000099107 (2011).

182 Bravo, J. A. et al. Ingestion of Lactobacillus strain regulates emotional behavior and central GABA receptor expression in a mouse via the vagus nerve. Proc Natl Acad Sci U S A 108, 16050-16055, doi:10.1073/pnas.1102999108 (2011).

183 Stephen, A. M., Wiggins, H. S. \& Cummings, J. H. Effect of changing transit time on colonic microbial metabolism in man. Gut 28, 601-609 (1987).

184 Vecsei, L., Szalardy, L., Fulop, F. \& Toldi, J. Kynurenines in the CNS: recent advances and new questions. Nat Rev Drug Discov 12, 64-82, doi:10.1038/nrd3793 (2013).

185 Sekine, A., Kuroki, Y., Urata, T., Mori, N. \& Fukuwatari, T. Inhibition of Large Neutral Amino Acid Transporters Suppresses Kynurenic Acid Production Via Inhibition of Kynurenine Uptake in Rodent Brain. Neurochem Res 41, 2256-2266, doi:10.1007/s11064-016-1940-y (2016).

186 Amori, L. et al. Specific inhibition of kynurenate synthesis enhances extracellular dopamine levels in the rodent striatum. Neuroscience 159, 196-203, doi:10.1016/j.neuroscience.2008.11.055 (2009).

187 Li, Y., Xiang, Y.-Y., Lu, W.-Y., Liu, C. \& Li, J. A novel role of intestine epithelial GABAergic signaling in regulating intestinal fluid secretion. doi:10.1152/ajpgi.00497.2011 (2012).

188 Lameris, A. L. et al. Segmental transport of $\mathrm{Ca2}+$ and $\mathrm{Mg} 2+$ along the gastrointestinal tract. doi:10.1152/ajpgi.00093.2014 (2015).

189 Geier, E. G. et al. Structure-based ligand discovery for the Large-neutral Amino Acid Transporter 1, LAT-1. Proc Natl Acad Sci U S A 110, 5480-5485, doi:10.1073/pnas.1218165110 (2013). 
190 Duelli, R., Enerson, B. E., Gerhart, D. Z. \& Drewes, L. R. Expression of large amino acid transporter LAT1 in rat brain endothelium. J Cereb Blood Flow Metab 20, 1557-1562, doi:10.1097/00004647-200011000-00005 (2000).

191 Zhang, Y. et al. in J Neurosci Vol. 34 11929-11947 (2014).

192 Guidetti, P. et al. Astrocytic localization of kynurenine aminotransferase II in the rat brain visualized by immunocytochemistry. Glia 55, 78-92, doi:10.1002/glia.20432 (2016).

193 Hilmas, C. et al. The Brain Metabolite Kynurenic Acid Inhibits a7 Nicotinic Receptor Activity and Increases Non-a7 Nicotinic Receptor Expression: Physiopathological Implications. (2001).

194 Alkondon, M. et al. Targeted Deletion of the Kynurenine Aminotransferase II Gene Reveals a Critical Role of Endogenous Kynurenic Acid in the Regulation of Synaptic Transmission via a7 Nicotinic Receptors in the Hippocampus. doi:10.1523/JNEUROSCI.5631-03.2004 (2004).

195 Varga, D. et al. Systemic L-Kynurenine sulfate administration disrupts object recognition memory, alters open field behavior and decreases c-Fos immunopositivity in C57BI/6 mice. Frontiers in Behavioral Neuroscience 9, doi:10.3389/fnbeh.2015.00157 (2015).

196 DeAngeli, N. E. et al. Exposure to Kynurenic Acid during Adolescence Increases Sign-Tracking and Impairs Long-Term Potentiation in Adulthood. Frontiers in Behavioral Neuroscience 8, doi:10.3389/fnbeh.2014.00451 (2015).

197 Gundersen, V., Storm-Mathisen, J. \& Bergersen, L. H. Neuroglial Transmission. Physiol Rev 95, 695-726, doi:10.1152/physrev.00024.2014 (2015).

198 Derecki, N. C. et al. Regulation of learning and memory by meningeal immunity: a key role for IL-4. J Exp Med 207, 1067-1080, doi:10.1084/jem.20091419

jem.20091419 [pii] (2010).

199 Brynskikh, A., Warren, T., Zhu, J. \& Kipnis, J. Adaptive immunity affects learning behavior in mice. Brain Behav Immun 22, 861-869, doi:10.1016/j.bbi.2007.12.008

S0889-1591(07)00341-8 [pii] (2008).

200 Kipnis, J., Cohen, H., Cardon, M., Ziv, Y. \& Schwartz, M. T cell deficiency leads to cognitive dysfunction: implications for therapeutic vaccination for schizophrenia and other psychiatric conditions. Proc Natl Acad Sci U S A 101, 8180-8185, doi:10.1073/pnas.0402268101 (2004).

201 Wolf, S. A. et al. CD4-positive T lymphocytes provide a neuroimmunological link in the control of adult hippocampal neurogenesis. J Immunol 182, 3979-3984, doi:10.4049/jimmunol.0801218

182/7/3979 [pii] (2009). 
202 Radjavi, A., Smirnov, I., Derecki, N. \& Kipnis, J. Dynamics of the meningeal CD4(+) T-cell repertoire are defined by the cervical lymph nodes and facilitate cognitive task performance in mice. Mol Psychiatry 19, 531-533, doi:10.1038/mp.2013.79 (2014).

203 Hill, A. S., Sahay, A. \& Hen, R. Increasing Adult Hippocampal Neurogenesis is Sufficient to Reduce Anxiety and Depression-Like Behaviors. Neuropsychopharmacology : official publication of the American College of Neuropsychopharmacology 40, 2368-2378, doi:10.1038/npp.2015.85 (2015).

204 Malberg, J. E., Eisch, A. J., Nestler, E. J. \& Duman, R. S. Chronic Antidepressant Treatment Increases Neurogenesis in Adult Rat Hippocampus. (2000).

205 Drew, L. J., Fusi, S. \& Hen, R. Adult neurogenesis in the mammalian hippocampus: Why the dentate gyrus? , doi:10.1101/lm.026542.112 (2013).

206 Valley, M. T., Henderson, L. G., Inverso, S. A. \& Lledo, P.-M. Adult Neurogenesis Produces Neurons with Unique GABAergic Synapses in the Olfactory Bulb. doi:10.1523/jneurosci.2845-13.2013 (2013).

207 Derecki, N. C., Quinnies, K. M. \& Kipnis, J. Alternatively activated myeloid (M2) cells enhance cognitive function in immune compromised mice. Brain Behav Immun 25, 379-385, doi:10.1016/j.bbi.2010.11.009 (2011).

208 Munford, R. S. Endotoxemia-menace, marker, or mistake? J Leukoc Biol, doi:10.1189/jlb.3RU0316-151R (2016).

209 Grabert, K. et al. Microglial brain region-dependent diversity and selective regional sensitivities to aging. Nature neuroscience 19, 504-516, doi:doi:10.1038/nn.4222 (2016).

210 Levenstein, S. et al. Stress and exacerbation in ulcerative colitis: a prospective study of patients enrolled in remission. Am J Gastroenterol 95, 1213-1220, doi:10.1111/j.1572-0241.2000.02012.x (2000).

211 Goodhand, J. R. et al. Mood disorders in inflammatory bowel disease: relation to diagnosis, disease activity, perceived stress, and other factors. Inflamm Bowel Dis 18, 2301-2309, doi:10.1002/ibd.22916 (2012).

212 Ananthakrishnan, A. N. et al. Association between depressive symptoms and incidence of Crohn's disease and ulcerative colitis: results from the Nurses' Health Study. Clin Gastroenterol Hepatol 11, 57-62, doi:10.1016/j.cgh.2012.08.032 (2013).

213 Parrott, J. M., Redus, L. \& O'Connor, J. C. Kynurenine metabolic balance is disrupted in the hippocampus following peripheral lipopolysaccharide challenge. Journal of Neuroinflammation 13, 124, doi:10.1186/s12974-016-0590-y (2016).

214 Dantzer, R., The University of Texas, M. A. C. C. \& rdantzer@mdanderson.org. Role of the Kynurenine Metabolism Pathway in Inflammation-Induced Depression: Preclinical Approaches. 1-22, doi:10.1007/7854_2016_6 (2016). 
215 Pollak, Y. et al. Behavioral aspects of experimental autoimmune encephalomyelitis. J Neuroimmunol 104, 31-36 (2000).

216 Mohr, D. C. et al. Treatment of Depression Improves Adherence to Interferon Beta$1 \mathrm{~b}$ Therapy for Multiple Sclerosis. Archives of Neurology 54, 531-533, doi:10.1001/archneur.1997.00550170015009 (2016).

217 Marrie, R. A. et al. Differences in the burden of psychiatric comorbidity in MS vs the general population. doi:10.1212/WNL.0000000000002174 (2015).

218 Mishra, M. K. \& Yong, V. W. Myeloid cells - targets of medication in multiple sclerosis. Nat Rev Neurol 12, 539-551, doi:10.1038/nrneurol.2016.110 (2016).

219 Longbrake, E. E., Department of Neurology, W. U., St Louis, Missouri, Cross, A. H. \& Department of Neurology, W. U., St Louis, Missouri. Effect of Multiple Sclerosis Disease-Modifying Therapies on B Cells and Humoral Immunity. JAMA Neurology 73, 219-225, doi:10.1001/jamaneurol.2015.3977 (2016).

220 Platten, M. et al. Treatment of autoimmune neuroinflammation with a synthetic tryptophan metabolite. Science 310, 850-855, doi:10.1126/science.1117634 (2005).

221 Fischer, C. W. et al. Interferon-alpha treatment induces depression-like behaviour accompanied by elevated hippocampal quinolinic acid levels in rats. Behav Brain Res 293, 166-172, doi:10.1016/j.bbr.2015.07.015 (2015).

222 Yan, Y. et al. IDO upregulates regulatory T cells via tryptophan catabolite and suppresses encephalitogenic $T$ cell responses in experimental autoimmune encephalomyelitis. J Immunol 185, 5953-5961, doi:10.4049/jimmunol.1001628 (2010).

223 Xu, H., Zhang, G. X., Ciric, B. \& Rostami, A. IDO: a double-edged sword for $\mathrm{T}(\mathrm{H}) 1 / \mathrm{T}(\mathrm{H}) 2$ regulation. Immunol Lett 121, 1-6, doi:10.1016/j.imlet.2008.08.008 (2008).

224 Lovelace, M. D. et al. Current Evidence for a Role of the Kynurenine Pathway of Tryptophan Metabolism in Multiple Sclerosis. Front Immunol 7, 246, doi:10.3389/fimmu.2016.00246 (2016).

225 Birey, F. et al. Genetic and Stress-Induced Loss of NG2 Glia Triggers Emergence of Depressive-like Behaviors through Reduced Secretion of FGF2. Neuron 88, 941-956, doi:10.1016/j.neuron.2015.10.046 (2015).

226 Keirstead, H. S. \& Blakemore, W. F. The role of oligodendrocytes and oligodendrocyte progenitors in CNS remyelination. Adv Exp Med Biol 468, 183197 (1999).

227 Aharoni, R. et al. Demyelination arrest and remyelination induced by glatiramer acetate treatment of experimental autoimmune encephalomyelitis. Proc Natl Acad Sci U S A 105, 11358-11363, doi:10.1073/pnas.0804632105 (2008). 
228 Bergner, C. L. et al. Mouse models for studying depression-like states and antidepressant drugs. Methods Mol Biol 602, 267-282, doi:10.1007/978-1-60761058-8_16 (2010).

229 Magoc, T. \& Salzberg, S. L. FLASH: fast length adjustment of short reads to improve genome assemblies. Bioinformatics 27, 2957-2963, doi:10.1093/bioinformatics/btr507 (2011).

230 Caporaso, J. G. et al. QIIME allows analysis of high-throughput community sequencing data. Nat Methods 7, 335-336, doi:10.1038/nmeth.f.303 (2010).

231 Ahn, Y. T. et al. Characterization of Lactobacillus acidophilus isolated from piglets and chicken. Asian-Australasian Journal of Animal Sciences 15, 1790-1797 (2002).

232 Geem, D., Medina-Contreras, O., Kim, W., Huang, C. S. \& Denning, T. L. Isolation and characterization of dendritic cells and macrophages from the mouse intestine. $J$ Vis Exp, e4040, doi:10.3791/4040 (2012).

233 Xia, J. \& Wishart, D. S. Web-based inference of biological patterns, functions and pathways from metabolomic data using MetaboAnalyst. Nat Protoc 6, 743-760, doi:10.1038/nprot.2011.319 (2011).

234 Thellin, O. et al. Housekeeping genes as internal standards: use and limits. $J$ Biotechnol 75, 291-295 (1999).

235 Liu, C. M. et al. BactQuant: an enhanced broad-coverage bacterial quantitative real-time PCR assay. BMC Microbiol 12, 56, doi:10.1186/1471-2180-12-56 (2012) 


\section{Selected Publications}

Marin IA, Goertz J, Ren T, Rich S, Onengut-Gumuscu S, Farber E, Wu M, Overall CC, Kipnis J, and Gaultier A. (2017). Microbiota alteration is associated with the development of stress-induced despair behavior. Sci Rep 7,43859. doi:10.1038/srep43859

Marin, I. A. \& Kipnis, J. (2017). Central Nervous System: (Immunological) Ivory Tower or Not?. Neuropsychopharmacology 42(1): 28-35. doi:10.1038/npp.2016.122

Marin, I. \& Kipnis, J. (2013). Learning and memory ... and the immune system. Learn Mem 20, 601-606, doi:10.1101//m.028357.112 